\title{
A Computational Workflow for Generating A Voxel-Based Design Approach Based on Subtractive Shading Envelopes and Attribute Information of Point Cloud Data
}

\author{
Miktha Farid Alkadri ${ }^{1, *(D)}$, Francesco De Luca ${ }^{2}\left(\mathbb{D}\right.$, Michela Turrin ${ }^{1}\left(\mathbb{D}\right.$ and Sevil Sariyildiz ${ }^{1}$ \\ 1 Department of Architecture and Engineering Technology, Faculty of Architecture and the Built Environment, \\ Delft University of Technology, Julianalaan 134, 2628 BL Delft, The Netherlands; M.Turrin@tudelft.nl (M.T.); \\ I.S.Sariyildiz@tudelft.nl (S.S.) \\ 2 Department of Civil Engineering and Architecture, Tallinn University of Technology, Ehitajate tee 5, \\ 19086 Tallinn, Estonia; francesco.deluca@taltech.ee \\ * Correspondence: M.F.Alkadri@tudelft.nl
}

Received: 21 July 2020; Accepted: 7 August 2020; Published: 9 August 2020

check for updates

\begin{abstract}
This study proposes a voxel-based design approach based on the subtractive mechanism of shading envelopes and attributes information of point cloud data in tropical climates. In particular, the proposed method evaluates a volumetric sample of new buildings based on predefined shading performance criteria. With the support of geometric and radiometric information stored in point cloud, such as position (XYZ), color (RGB), and reflection intensity (I), an integrated computational workflow between passive design strategy and 3D scanning technology is developed. It aims not only to compensate for some pertinent aspects of the current 3D site modeling, such as vegetation and surrounding buildings, but also to investigate surface characteristics of existing contexts, such as visible sun vectors and material properties. These aspects are relevant for conducting a comprehensively environmental simulation, while averting negative microclimatic impacts when locating the new building into the existing context. Ultimately, this study may support architects for taking decision-making in conceptual design stage based on the real contextual conditions.
\end{abstract}

Keywords: voxel-design approach; shading envelopes; point cloud data; computational design method; passive design strategy

\section{Introduction}

\subsection{General Background}

The rapid development of 3D laser scanning technology has reached across multiple-disciplines within design and engineering. However, the practical implementation of this technology is often applied in major fields, such as photogrammetry [1,2], cultural heritage [3-6], and environmental engineering $[7,8]$. Digital reconstruction as one of the main subjects in these scopes has been used predominantly for building performance assessments $[9,10]$, where the contextual modeling of existing studies is frequently based on a 3D solid modeling context [11,12]. As a consequence, high computational costs and time are required to cover the entire set of complex building forms. On the other hand, the use of point clouds during the early stage of architectural design has not yet been fully explored, especially related to the performance simulation task and design decision support.

As an entity of 3D data scanning, Otepka et al. [13] illustrates the point cloud as a universal denominator for laser scanning and photogrammetric data. Its data structure is principally characterized 
by position information (XYZ) as a permanent element coupled with auxiliary information attached to it, such as color attributes (RGB), reflection intensity (I), and any abstract information [14]. The prospective applications of these attributes not only represent metadata information of the real environment, but also enable designers and researchers to perform numerous tasks, such as data processing, visualization, and analysis. Moreover, this can help architects further to address environmental design issues, such as solar and shading performance.

The technological advancement of point cloud reveals the relevance of integrating it into the passive design strategy, especially when dealing with generative architecture designs that currently lack several relevant aspects. For example, first, understanding the site characteristics of an existing environment. While 3D site modeling primarily deals with a building-oriented context, surrounding properties, such as vegetation and adjacent buildings, are often neglected [15]. This may not only affect the performance simulation of a proposed design, but also potentially create microclimatic issues when it comes to the real context. Second, the absence of surface properties, such as roughness and material characteristics on a manually-built 3D model, may cause a crucial discrepancy when dealing with environmental simulation between planned and existing buildings [16]. With geometric and radiometric information extracted from point cloud data, this study, therefore, proposes an integrated passive design approach based on shading performances of new and existing contexts.

As a contextual design approach, this study specifically investigates the idea of subtractive shading envelopes that are principally extracted from the concept of solar envelopes initially introduced by Knowles [17]. In this regards, solar envelopes permit architects to design appropriate massing of a new building into the existing environment by guaranteeing desirable sun access for surrounding buildings during the critical period [18], while subtractive shading approach aims to extract potential performances of the existing contexts and integrate it with a 3D volumetric massing of a proposed building based on predefined shading performance criteria.

Since then, various computational methods of solar envelopes, such as descriptive geometry, solar obstruction angle, and constructive solid geometry have been defined [19]. These approaches have successfully demonstrated the concept of solar envelopes into various urban settings (e.g., single building, open space, and urban scale) and multiple functional utilities (e.g., housing, offices, and commercial buildings). It is worth noting that the contextual settings of the existing methods primarily focus on temperate zones of southern and northern hemisphere countries, which have distinct climatic conditions during the four-seasons. This means that design objectives and climatic parameters of most existing methods for solar envelopes become less applicable when it comes to tropical countries, especially for those located on the Equator, such as Indonesia. Since tropical countries present wet and dry seasons all year round, the objective of solar envelopes significantly shifts and aims to minimize the penetration of direct sun access to the buildings, due to high temperatures. For example, housing in Indonesia is typically designed in a way that prohibits direct sunlight from penetrating the dwelling, especially into primary living spaces, so that temperatures are kept low during the day. Consequently, the air conditioner (AC) frequently becomes a short-term solution to mitigate the building's temperature, which unfortunately contributes to the annual increase in energy consumption [20]. Accordingly, shading conditions become considerably relevant for urban forms generation in tropical contexts. This study specifically proposes an environmental design strategy that integrates shading performance aspects and attributes information from point cloud data through a computational workflow of a voxel-based design approach.

Furthermore, the following section will present a theoretical background of the existing studies, starting from solar envelopes, shading envelopes, subtractive solar envelopes, and subtractive solar envelopes based on point cloud data so-called SOLEN approach. This will be followed with a description of a proposed method in Section 2, while case studies in Section 3. Section 4 will comprehensively discuss the findings of the simulation results. Lastly, Section 5 will describe the conclusions, limitations, and future recommendations of the study. 


\subsection{Related Works}

\subsubsection{Solar Envelopes}

In the remote past, the concept of vernacular architecture has successfully contributed to preserving sustainable building envelopes [21,22]. This can be observed through the development of the Indus Valley, Mohenjo-Daro in India, 2500 BC [23], El-Lahun village in Egypt (1857-1700 BC) [24], and many classical Greek cities, such as Olynthus in North Hill-a city designed to benefit from passive solar energy for the heating of buildings [25]. This strategy was known as solar-oriented homes or so-called "solar architecture". Since then, solar architecture has become an essential guide for designers to develop sustainable urban planning. For example, Andrea Palladio has discussed the proper norms of city planning by considering wide streets for cold climate countries and narrow streets for tropical countries [26]. Additionally, Ildefons Cerdà integrated green areas into the public and private space of Barcelona in his masterplan of the city so as to enhance the comfort of inhabitants [27]. During the industrial revolution, the idea of urban solar policy or refers to the post-war housing was also implemented in France (in 1912), Germany (in 1920), and New York (in 1916). These examples have shown a positive contribution to architectural buildings, not only to reduce the energy consumption of the built environment [28], but more importantly, to support a healthy living environment [29]. Furthermore, the idea of solar accessibility has been elaborated further through the concept of solar envelopes.

By definition, solar envelopes stand for imaginary boundaries that are constructed based on the sun's movement. It is regulated based on specific space-time constraints [18]. According to this principle, solar envelopes can be transformed into geographic and climatic properties within the size of on-site buildings [19]. Geographic properties deal with a group of parameters that define the spatial relationship between the design plot and existing context related to orientation typology, surrounding facades, sidewalks, building height, longitude, latitude, floor area ratio (FAR), setback, shadow fences, and street sizes. On the other hand, climatic properties consist of parameters that determine the geometric transformation of the proposed building based on the time construction, such as cut-off-times, solar angle, sun path, dry bulb temperature, sun access hours, solar altitude, and solar azimuth. These parameters are used not only to generate solar envelopes, but also to identify the character and qualities of the built environment. For example, orientation plays a great role in examining the geometrical shape of solar envelopes, especially when dealing with the street layout in relation to various angular values, colonnades with a variety of direct solar radiations, and solar urban layouts [30]. A seasonal leaf cover from the surrounding vegetation can also affect the geometrical configuration of solar envelopes as it may be considered as a part of geographic elements for violating excessive direct sun access during summer [16]. Besides, the solar angle as a climatic property is used to determine geometric solar envelopes based on the construction planes [31]. It is mostly employed for simple shape plots, with borders aligned with the main cardinal directions in east-west (EW) and north-south (NS) and for the main hours and days, such as the noontime during summer and winter solstice, and spring/autumn equinoxes.

\subsubsection{Shading Envelopes}

As opposed to solar envelopes, the concept of shading envelopes primarily deals with the solar radiation-reduction to achieve appropriate daylight for urban equatorial climates. This permits architects not only to establish a geometrical configuration of solar shading envelopes, but also to control the direct sun exposure of the building's own façades and surroundings during a critical time. Two different types of shading approaches are identified as follows:

- $\quad$ Building forms

In this part, the concept of shading envelopes aims to promote a passive design strategy through the form generation within the conceptual design stage. This means that the volumetric shape of 
proposed buildings is developed based on the consideration of solar shading criteria. An interesting example can be observed through the concept of "shadow umbrella" introduced by Emmanuel [32]. This concept proposed a design approach of shading strategies incorporated with natural elements, such as vegetation and water bodies, aiming to create shading for adjacent buildings and to mitigate the urban heat island for tropical neighborhood areas. Accordingly, a new configuration of urban block shapes with a thermally comfortable can be generated. DeKay [33] addressed a similar strategy with the concept of climatic envelopes. The climatic envelope primarily aims to generate a building mass that guarantees access for diffused daylight and solar energy resources for the surrounding buildings. This concept specifically contains a geometrical intersection between daylight and solar envelopes based on the sky exposure plane and solar protection plane, respectively. In this case, sky exposure plane refers to imaginary sloping planes that allow penetration of natural light and air on the building facades in higher density districts [34]; meanwhile, solar protection plane refers to an inclined plane that is generated from the profile angle, the so-called vertical shadow angle (VSA) [35]. In a similar vein, Capeluto [36] proposed the concept of self-shading envelopes by extending the functional properties of the sky exposure plane through the solar collection envelopes (SCE) model. The geometrical configuration of self-shading envelopes results in cone shapes, due to the required façade inclination and shading orientation. Therefore, the envelope's roof areas should be larger than the bottom part of the envelope geometries. This approach aims to avoid overheating and at the same time, to maximize the self-building protection for a certain period during summer.

- $\quad$ Building components

In addition to building forms, shading approaches are also applied to specific building components, such as windows, cantilevers, and openings on the building façade, based on the determined building shapes. Although this study limits the scope of investigation on a form-finding design solution, some studies on shading mechanisms drive potential efforts to handle more complex projects. For example, Yezioro and Shaviv [37] proposed SHADING as a design and evaluation tool for analyzing mutual shading between buildings and other surrounding properties, such as vegetation. It specifically calculates the insolated fraction on the building surfaces quantitatively and performs a ray-tracing algorithm to identify the shadows visually at a particular time. Similarly, Marsh [38] also used a ray-tracing analysis to identify the external obstruction of solar intensity on the optimized shape of shading geometries. In this case, optimized shading designs have effectively accommodated passive solar control through the building apertures [39,40] Other approaches deal with a graphic solution of shading design tools [41], form-finding of static exterior shading devices called SHADERADE [42], and a cellular method to define optimal shading patterns [43].

Although these approaches may address the aspect of tropical design contexts, they lack some critical aspects during the simulation of shading envelopes. First, the quality of solar radiation (i.e., the quantity of direct sunlight hours) received by surrounding buildings is not taken into account by most methods and tools, due to a fixed period when determining direct sun access. Consequently, all geometrical shapes of existing buildings are treated similarly when receiving the irradiation qualities without considering the obstruction of properties and building orientation of the plot. Second, shadow fences of surrounding buildings' facades are primarily regulated by a Z-axis. This makes the design configuration of the resulting envelopes rely only on the horizontal shading lines. In fact, shading areas of surrounding façades are more complex, especially when dealing with dense areas and multiple urban forms. In order to compensate for these issues, the existing studies present some relevant aspects, such as subtractive mechanism and point cloud data that may be useful for further development. This will be discussed in the following section. 


\subsubsection{Subtractive Solar Envelopes}

As previously mentioned in the general background, this approach specifically subtracts a volumetric matrix of the 3D plot according to solar accessibility criteria. This is done by projecting solar vectors acquired from the number of direct sunlight hours on surrounding building facades. In principle, this mechanism has been addressed by Leide and Schlüter [44,45] via volumetric site analysis (VSA). Their approaches aim to explore urban site information by simulating multiple environmental performances, such as solar radiation, airflow, visibility, thermal comfort, and wind velocity through volumetric insolation analysis (VIA), volumetric visibility analysis (VVA), and computational fluid dynamics (CFD). Such this approach and other related developments [46-48], however, merely focus on the architectural form-finding without any further consideration on the concept and design principles of solar or shading envelopes.

On the other hand, De Luca [49-51] and Darmon [52] have proposed a similar subtractive mechanism based on the performance criteria of solar envelopes or so-called subtractive solar envelopes. This approach specifically involves sun visibility that aims to evaluate sun vectors from a predefined shadow grid of surrounding building windows without any obstruction from other existing buildings. In parallel, ray-tracing analysis is performed from surrounding windows to the voxels within the 3D plot using a Boolean expression (true or false statement). In this operation, the true statement will be executed when sun vectors hit or intersect the 3D polyhedra, and accordingly, the voxels subtraction procedure to the 3D polyhedra can be performed. Meanwhile, the false statement indicates an unsuccessful intersection. This condition means that voxels that are not intersected may contribute to the generation of geometric solar envelopes.

The subtractive solar envelopes ultimately permit architects not only to deal with various geometric configurations based on solar performance analysis, but also to highlight the potential use of voxel-based generative designs for urban environments. However, despite such potential improvements, aspects (such as sun visibility and ray-tracing analysis) pose several critical considerations, especially when addressing the contextual design strategy. For example, the identification of visible sun vectors merely considers the surrounding building contexts while neglecting relevant geometric properties, such as vegetation and other site characteristics (e.g., material properties). This consequently can affect irradiation analysis during the environmental performance simulation between a proposed building and the existing contexts. Besides, the window's grid configuration lacks in representing the insolation values of building facades during the ray-tracing analysis, due to its limited consideration of geometric centroids of each surrounding window.

In order to address these gaps, the existing workflow of subtractive solar envelopes has been improved by incorporating it with the prospective application of 3D laser scanning (point cloud data). By exploiting the practical usability of the point cloud in different fields, the scope of information properties in the real contexts can be improved, and to some extent, it becomes relevant to the specific aforementioned issues. Regarding sun visibility, the 3D point cloud not only captures the most and the least sun-exposed areas through buildings and vegetation, but also investigating material performances of contextual datasets through optical (reflectivity and translucency) and thermal properties (albedo and emissivity). Besides, the ray-tracing analysis between a proposed building and surrounding contexts is performed based on 3D point cloud datasets of the existing context. In other words, it substitutes the surrounding window's grid on the building facades proposed by the existing approach.

\subsubsection{Subtractive Solar Envelopes Based on Point Cloud Data (SOLEN)}

With the support of geometric and radiometric properties [53,54] stored in a 3D point cloud, the integrated computational workflow between subtractive solar envelopes and attribute information of point cloud data has been established $[55,56]$. It specifically integrates functional properties of position information (XYZ), color information (RGB), and reflection intensity (I). Each of these attributes caters to different potential tasks. For example, color information (RGB) can be used not only to extract and segment certain areas within the dataset based on its values $[57,58]$, but also to translate them 
into new information properties [59,60]. This can include converting data attribution of RGB into HSV values to perform the measurement analysis and road maintenance [61] and extracting the semantic information of the indoor environment with automatic room labeling [62,63]. Meanwhile, reflection intensity (I) predominantly deals with surface and spectral properties of the scanned objects [64] as it constitutes the return strength values of laser pulse or backscattered echo for each recorded point [54]. Accordingly, the intensity values can be used not only to map geological layers and pavement lines [54], but also to detect natural phenomena, such as frozen and wet surfaces on roads [65], and the measurement of seasonal snow cover [66]. On the other hand, position information (XYZ) constitutes of geographic coordinate that marks each recorded point's specific location. This attribute plays a great role in synchronizing index between color and intensity values as it can attach to both attributes. Thus, complex areas of the dataset can be precisely extracted based on selected values. In general, these attributes contribute not only to extend particular performances of 3D point cloud data, such as identification of existing material properties [67], but also to extend the applicability of environmental analysis during the conceptual design stage.

Before performing the subtractive solar envelopes, the dataset correction is required to minimize erroneous levels during scanning. In this case, some aspects, such as environmental and meteorological conditions, atmospheric pollution [68,69], unit specification of the scanner, surface properties of the scanned objects, and scanning geometries [70] can principally affect metadata information of the datasets during scanning. While correcting all these variables seems impractical, due to some local constraints (i.e., manufacturers), this study specifically focuses on correcting the acquisition geometry based on the angle of incidence, which is relevant to the proposed subtractive mechanism. Having established the corrected datasets, it can be further used not only to perform the ray-tracing analysis between the 3D polyhedra and selected solar vectors, but also to calculate the material properties of existing contexts that are useful to evaluate the environmental performance of a proposed building. In parallel, insolation analysis is performed to identify the potential solar energy of the resulting solar envelopes.

\section{Proposed Methods for Subtractive Shading Envelopes}

This study proposes a computational framework that consists of three phases: input, simulation process, and output (see Figure 1). Within a simulation process, five sequential procedures are developed, ranging from A (input parameters) to E (form generation process of self-shading envelopes).

To perform specific tasks in each predefined step, the proposed workflow was supported by several digital tools. For example, Topcon GLS 2000 [71] was employed to collect high-resolution point cloud datasets. It was complemented with Maptek I-Site [72] to perform dataset registrations, coloring, modeling, and most importantly, to facilitate the data transfer from scanner to the workstation in any designated format. Moreover, Cloud Compare (CC) [73] was used, not only for dataset preparation, but also for dataset pre-processing, such as attribute selection, dataset formatting, scalar field features, and the normal surface calculation. In alignment with that, Matlab [74] was specifically used to assist dataset correction (i.e., optimal normal values, intensity correction, and dataset subsampling), while Rhino [75] (coupled with Grasshopper [76] for visual scripting) was employed to develop a 3D geometric model of a proposed building and to perform solar simulation analysis by using a Ladybug [77] component in Grasshopper. Furthermore, a detailed task, dataset formats, and outputs of each step are addressed below. 


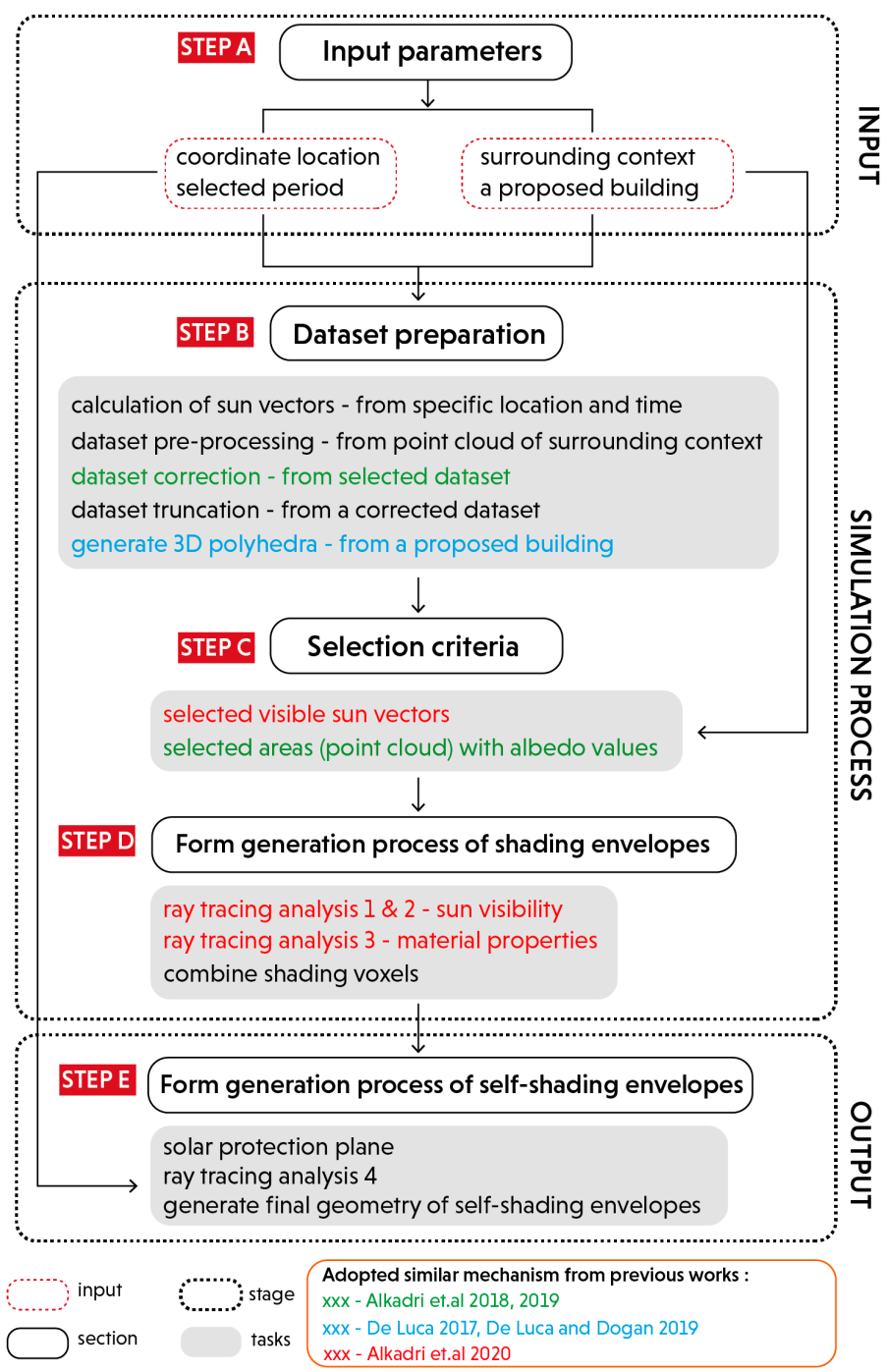

Figure 1. An overview of the proposed computational workflow.

\subsection{Stage A Input (Step A-Preparation of Input Parameters)}

As a starting point for the computational procedures, the input section refers to step A, which contains a series of parameters that are used to construct contextual settings of the subtractive shading envelopes. Specifically, it consists of climatic properties that correspond to coordinate location (i.e., longitude and latitude position based on World Geodetic System 1984, WGS84) and selected periods (i.e., month, year, day, and hours) (see Figure 2). The longitude and latitude coordinates influence the envelope's geometrical properties on the basis of sun position and solar angle. For example, high-latitude sites are characterized by a small angle of solar altitude and smaller degrees of solar radiation. Moreover, specific periods are required to obtain the number of critical hours of natural illumination that affects a proposed building and surrounding contexts.

On the other hand, geographic properties include surrounding contexts (i.e., existing buildings and vegetation) and a geometric model of the proposed building. In this regard, the surrounding context contains a 3D point cloud data of the existing environment. As a raw dataset, its format properties often rely on the type of 3D scanner, but as long as the required attribute information (i.e., $\mathrm{XYZ}$, RGB, and reflection intensity) is legible, any raw dataset formats are acceptable (i.e., PTX file). Meanwhile, several parameters, such as height, width, floors, setback, and building function, must be established to generate an initial 3D envelope for a proposed building. These inputs are then executed into the following Step B (dataset preparation) based on corresponding parameters and tasks. 


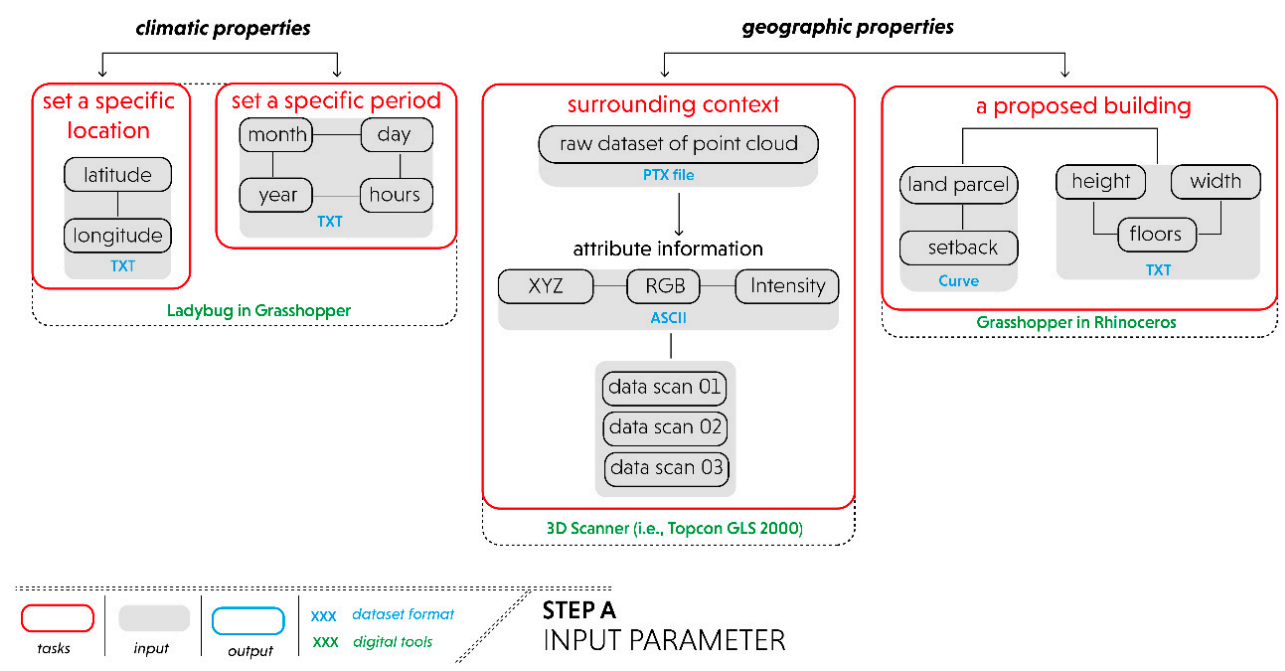

Figure 2. Preparation of input parameters.

\subsection{Stage B Simulation Process}

This section focuses on translating the raw datasets into a simulation model by examining three main steps (i.e., dataset preparation, selection criteria, and form generation process of shading envelopes). Each step serves specific actions that are performed sequentially based on specific computational tasks. As illustrated in Figure 1, this section adopts several workflows that are partially implemented from previous works. For example, pre-processing point cloud datasets and the calculation of material properties (i.e., albedo values) are indicated with a green text [67]. These works focused on developing a material database of existing contexts and solar radiation analysis based on a small sample of point cloud data. Next, 3D polyhedra and sun visibility analysis are illustrated in the blue text $[49,50]$. These works primarily investigated a voxel-based generative design of subtractive solar envelopes based on 3D and parametric modeling. Last, the ray-tracing analysis between a proposed building and point cloud data of surrounding contexts is illustrated in the red text [56]. This work refers to a form generation of subtractive solar envelopes that consider surface properties of existing contexts based on geometric and radiometric information of point cloud data. While these previous works address different objectives, some features are still relevant for supporting an integrated design concept and computational workflow for establishing the subtractive shading envelopes.

To illustrate specific tasks in this stage, a detailed discussion of each proposed step is presented below.

\subsubsection{Step B-Dataset Preparation}

This step aims to prepare all the necessary datasets to be readily used in the simulation model. After establishing the input from climatic and geographic properties, five tasks are required to perform. Two of these tasks (i.e., sun vectors calculation and the initial envelopes generation) can be run in parallel while the other three tasks (i.e., pre-processing datasets, dataset correction, and subsampling dataset) can be executed sequentially (see Figure 3).

Task 1, sun vectors refer to the number of sunlight hours that must be preserved on surrounding facades during the required period. As compared with cut-off times, which refer to a fixed period, sun access duration can be selected from a range of available hours for a specific façade on a specific day or for each day during a specific period. In doing so, sun visibility plays a crucial role when determining the relevant sun vectors.

Task 2, the initial envelope of a proposed building, is generated based on the predefined criteria. In this case, the functional program of a proposed building is projected as a public library, as well as communal space for the local community. To support the main activities, some spaces are established, such as a reading room, meeting room, toilet, and exhibition areas. Accordingly, the building needs 
to be accessible, and its indoor environment should be thermally comfortable for supporting the daily activities.

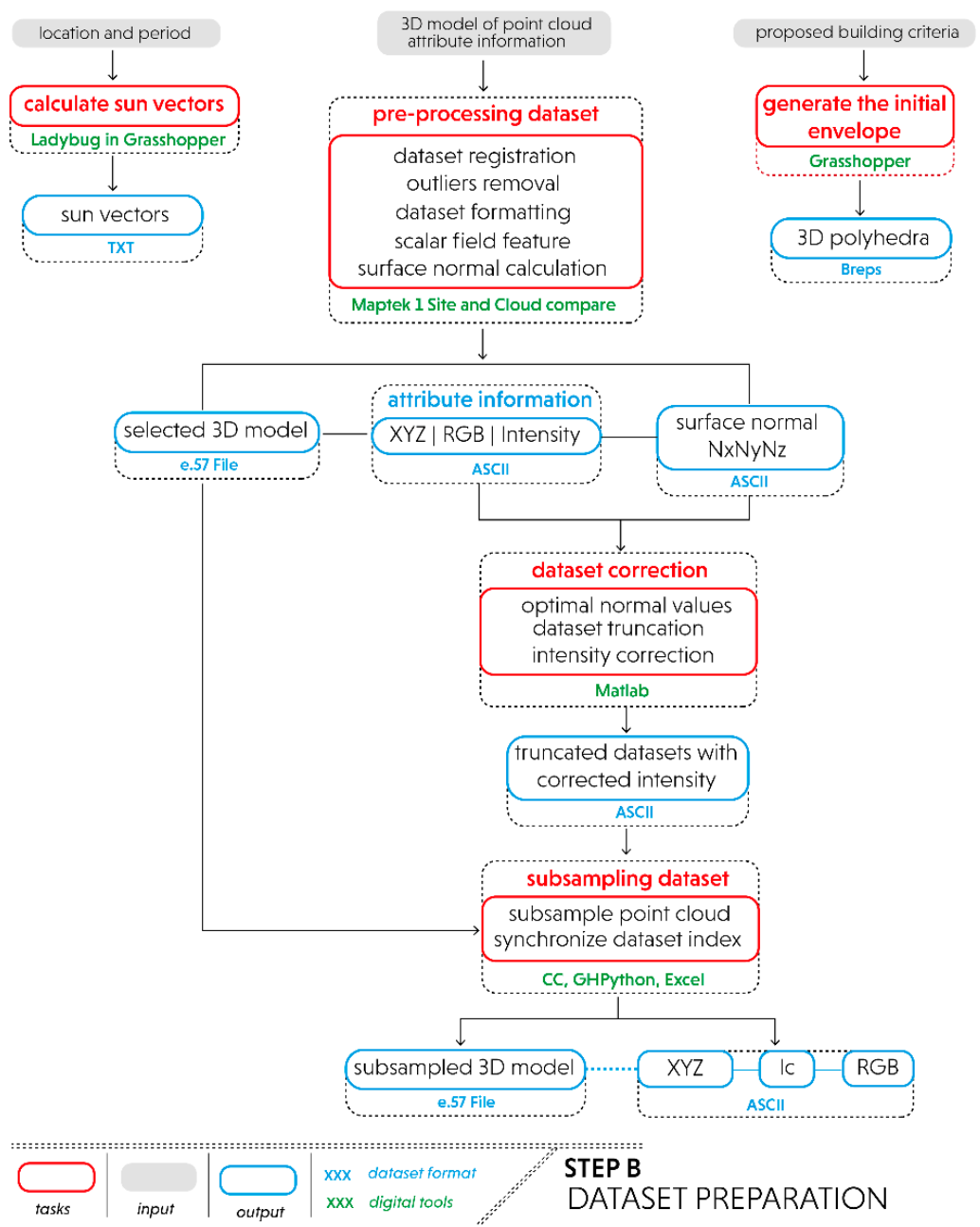

Figure 3. Detailed procedures for the dataset preparation.

Task 3, the pre-processing tasks start with the dataset registration. This process aims to locate recorded datasets into a standard coordinate system based on the reflector position and GPS orientation. Afterwards, the outlier (unnecessary cloud of points) removal is performed not only to clean the boundary of selected datasets, but also to filter noises created during scanning. The dataset formatting also plays an important role in compensating for interoperability issues during the simulation process. In this case, the initial format of 3D raw point clouds (i.e., PTX) and its metadata are converted to E57 and ASCII files, respectively, in order to be accessible for various digital processing tools. In addition to this, the scalar field of the dataset is activated to identify the attached values' scale in each attribute. Last, normal surfaces $(\mathrm{NxNyNz})$ of the dataset are calculated to find the appropriate normal values of each projected point during scanning. As normal values of the raw point cloud are excluded in the typical attribute properties, various angles of incidence, ranging from the sample of $10^{\circ}$ to $90^{\circ}$, are firstly computed to each data scan. This is done by using the Hough Normal plugin [78] in CC, due to the original form of unstructured raw point cloud data. In this regard, each point within each data scan has different preliminary normal values. Ultimately, task 3 results in several outputs, such as a 3D model of selected datasets, attribute information of point cloud data with raw intensity values, and the normal surface of each data scan at various incidence angles.

Task 4, the dataset correction, is performed to compensate for the scattering condition of unstructured point clouds during scanning, specifically for amending radiometric properties of 
the dataset. It is worth noting that this procedure can only be applied on a single scan, due to the potentiality of mixing a reference point of the scanner and intensity values on the merge datasets [54]. The correction step starts with finding the average distribution of optimal projection points from the preliminary normal values. This achieves a reliable normal surface on each applied angle in the data scan. To do so, the following equation [79] is applied with an assumption that the original position of a 3 D scanner located at $(0,0,0)$.

$$
i=\cos ^{-1}\left(\frac{\overline{d n} \cdot \overline{d l}}{|\overline{d n}||\overline{d l}|}\right)
$$

where:

$i=$ initial incidence angle

$\overline{d n}=$ direction of the normal surface

$\overline{d l}=$ direction of the laser pulse

After configuring the point distribution from a various range of cosinus products, an evaluation is conducted to the standard deviation of each registered cosine value within the dataset. It aims to identify the pattern of point density to reduce scattered and coarse point clouds through the dataset truncation. Afterwards, the truncated datasets can be used for the intensity correction. In this regard, the angle of incidence becomes a relevant factor for correcting the dataset's acquisition geometry, given that instrumental effects highly affect the raw intensity value of TLS datasets. This procedure is executed based on the following equation [54].

$$
I_{c}=I_{\text {raw }} \cdot \frac{1}{\cos \alpha}
$$

where:

$$
\begin{aligned}
& I_{c}=\text { corrected intensity } \\
& I_{\text {raw }}=\text { original intensity } \\
& \alpha=\text { angle of incidence }
\end{aligned}
$$

Task 5, the subsampling dataset, is performed in CC to reduce the density of points during the simulation. However, this procedure creates interoperability issues when the resulting dataset is matched and visualized to the initial 3D model in Rhino. Specifically, the 3D model cannot directly recognize the input of subsampled datasets, due to different units and scales of the attribute information. Therefore, synchronizing the initial index between the two datasets identifies metadata information in the geometric 3D model. This further permits the extraction of certain areas in the 3D dataset based on selected attributes values.

\subsubsection{Step C-Selection Criteria}

After preparing the corrected datasets from climatic and geographic properties, this study sets two environmental performance criteria that support the geometric generation: sun visibility and material properties (see Figure 4). Sun visibility plays a crucial role in filtering the sun vectors that have direct access to the dataset of surrounding contexts. To do so, sun vectors generated from the indicated period are multiplied with normal vectors extracted from subsampled datasets. The resulting normal irradiance values are then evaluated on the basis of the projected angle. In this case, irradiance values with equal and larger than $90^{\circ}$ are eliminated as they consist of zero and negative cosine values. Accordingly, these values are then excluded within the list of visible sun vectors because the surface of the datasets does not properly absorb their solar energy. Afterward, the resulting values can be used to select the corresponding points within the dataset.

On the other hand, material aspects are used to measure the performance behavior of the existing site's surface properties. This allows architects to identify susceptible areas that may affect the geometrical performance of the proposed design. This study specifically computes albedo values to detect the absorbance percentage of solar energy on surrounding contexts by considering the RGB color 
and corrected intensity (Ic) of contextual datasets. A detailed procedure regarding the calculation of albedo values can be found in our previous research [67]. Furthermore, the resulting albedo values are filtered based on the threshold below 0.3. Although this setting indicates the low albedo, it can be used not only to identify areas that contain a high level of heat absorbance, but also to analyze and mitigate microclimatic impacts of the surrounding areas especially related to thermal issues and urban heat island (UHI) effect. In order to avoid that, these areas need to be blocked from direct sun exposures by excluding the indicated surfaces with high albedo values. Afterward, the resulting indexes of low albedo values (below 0.3) are synchronized not only with selected normal vectors from the sun visibility to register the corresponding normal surface of the dataset, but also with XYZ attributes to select the matching points within the dataset.

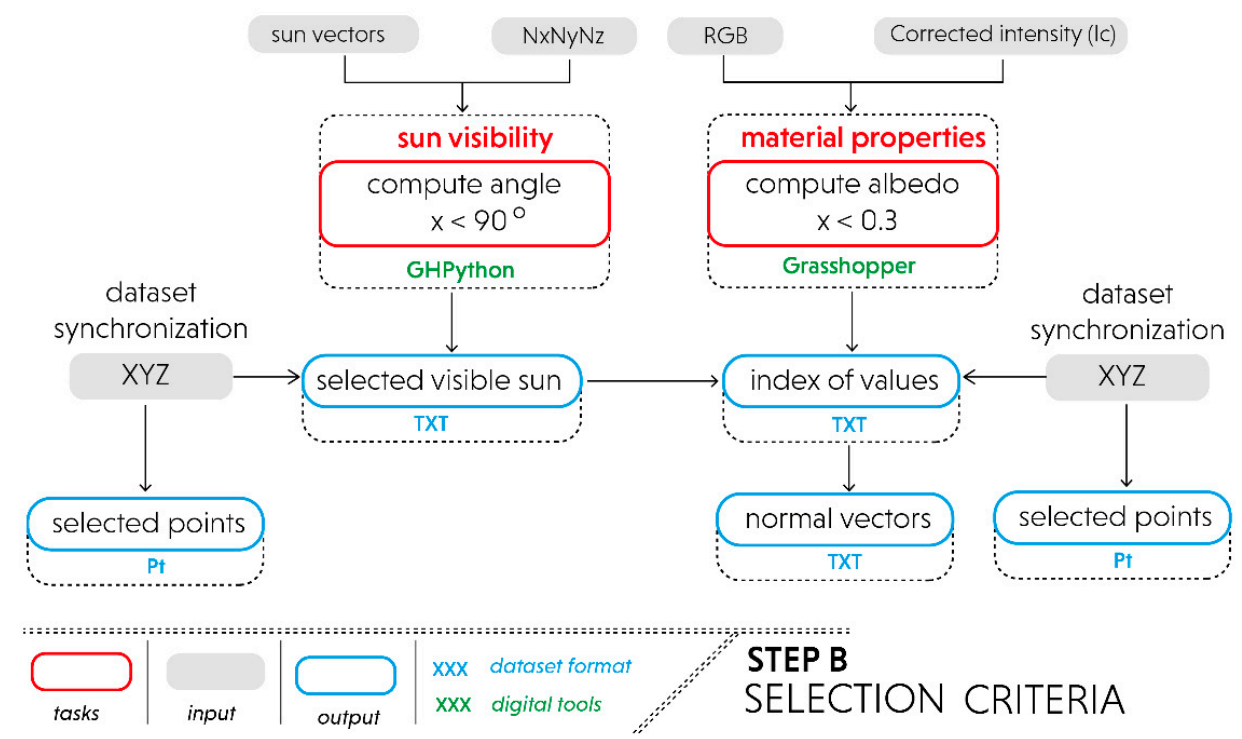

Figure 4. Selection procedures based on the criteria of sun visibility and material properties.

\subsubsection{Step D-Form Generation Process of Shading Envelopes}

After establishing all the required parameters from the selection criteria, this step focuses on developing the simulation workflow (see Figure 5). It starts with the first ray-tracing analysis that requires input from visible sun vectors, selected corresponding points from sun visibility, and 3D polyhedra of a proposed building. This procedure principally applies a Boolean expression to assign true or false conditions on selected voxels within the 3D polyhedra array. The ray-tracing analysis-01 generates intersecting rays that are then evaluated based on the predefined criteria of direct sun access. In this case, voxels that are not blocking sun access to the proposed buildings or are categorized as an unsuccessful intersection with the 3D polyhedra will be considered part of the shading voxels (refer to voxels-02 in Figure 5). This workflow can also be called as a reverse solar envelope. Meanwhile, voxels that receive sun access will be forwarded to a later step in the second ray-tracing analysis (refer to voxels-01 in Figure 5).

Furthermore, reference points are generated from the voxels- 02 to be used in the simulation of ray-tracing analysis-02. As a follow-up to the previous procedure, the ray-tracing analysis-02 aims to maximize the geometric generation of shading voxels. In doing so, by changing the basis projection of initial reference points originated from surrounding buildings to the voxels-02 may compensate for geometric obstruction of polyhedra at a certain projection angle in the ray-tracing analysis- 01 . Instead of applying this procedure to the original 3D polyhedra, it is used to re-evaluate voxels-01 based on reference points of voxels-02 so as to identify additional voxels (refer to voxels-03) that fulfill the criteria of receiving shading condition. As for the input for material properties, a similar procedure of ray-tracing analysis is also performed by considering the lowest albedo values (ranging from 0 to 0.3) applied to surround contexts. These results in voxels-04 so that in total, three groups of voxels 
(i.e., voxels-02, voxels-03, and voxels-04) are generated to shade surrounding buildings. These voxels are then combined into one group, voxels- 05 . To ensure that a shading condition also applies to a proposed building, the self-shading workflow is performed in the following stage.

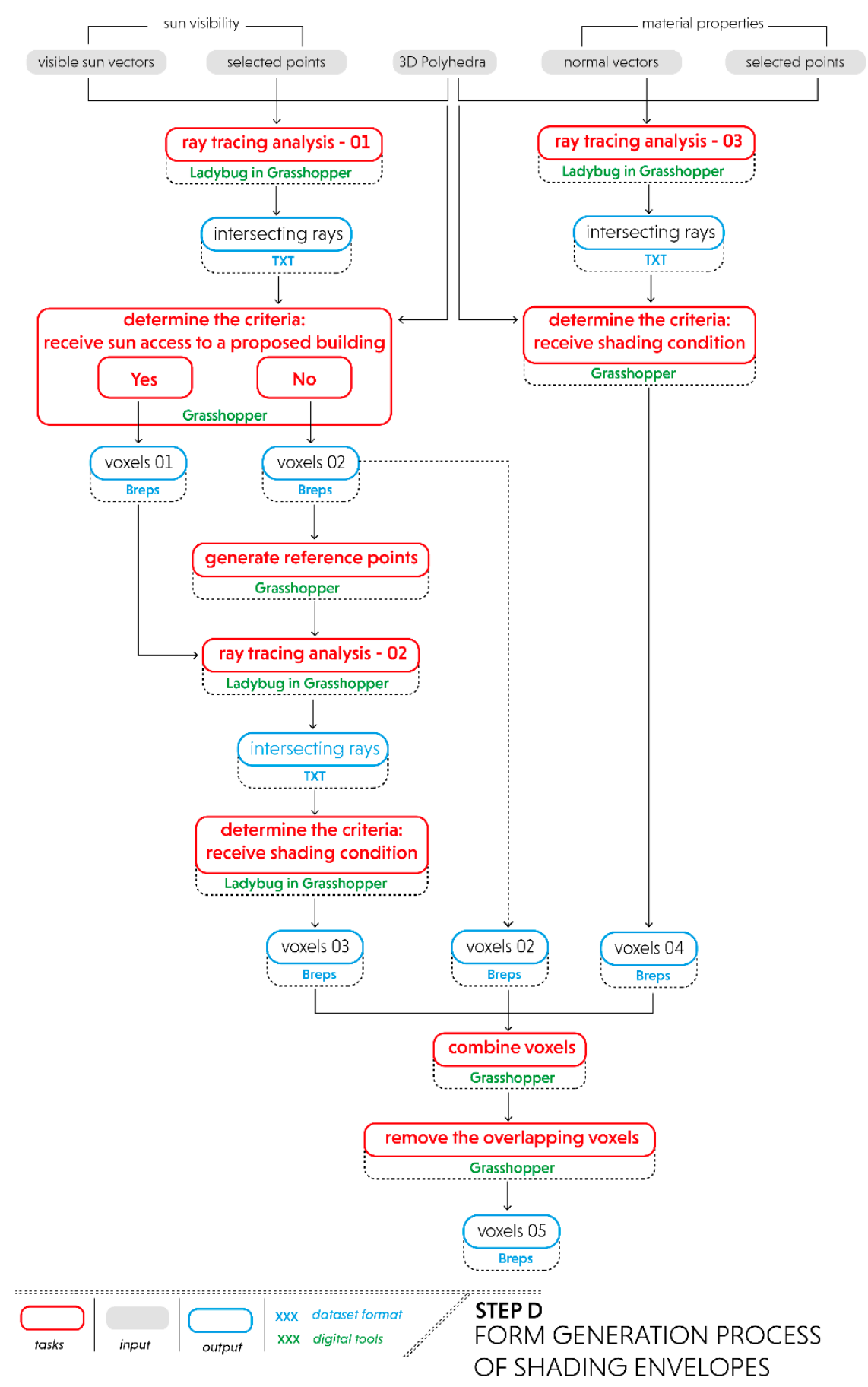

Figure 5. Detailed procedures for the design simulation.

\subsection{Stage C Output (Step E-Form Generation Process of Self-Shading Envelopes)}

As a last stage of the computational workflow, the output contains final tasks to generate a geometric configuration of self-shading envelopes (see Figure 6). The first task begins with selecting the upper part of the stacking voxels. This upper part acts as a roof or shelter to guarantee a shading condition for all properties under its envelope within a predefined period. Afterward, a solar protection plane can be applied on the bottom surfaces of the upper part of the voxels. It aims to establish 
reference points that are used as the basis for ray projections. Furthermore, the ray-tracing analysis-04 is performed by considering inputs from reference points of the upper voxels, the remaining stacking voxels (i.e., bottom part), and initial sun vectors calculated for the analysis period under consideration. This simulation will evaluate the remaining voxels by maintaining the one that receives a shading condition while removing the unshaded voxels. The resulting voxels (refer to voxels-06) are then combined with the upper voxels to establish the geometric envelope for each data scan (refer to voxels-07 and voxels-08). After identifying self-shading voxels for each data scan, the final step is to combine all these voxels into a final geometric envelope that represents a final configuration of envelopes (refers to voxels-09).

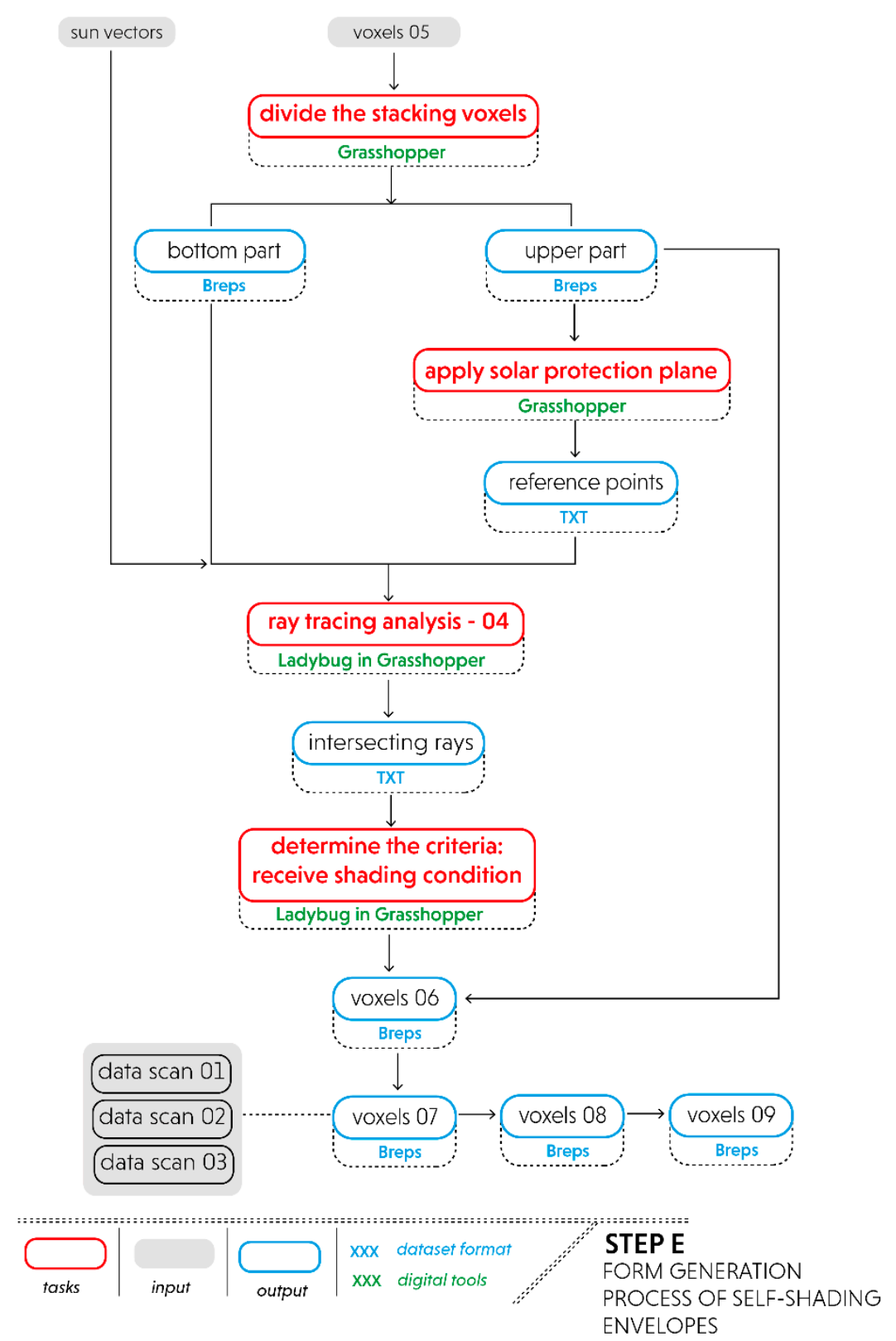

Figure 6. Detailed procedures for generating the final output of self-shading envelopes.

\section{Dataset Collection}

In regards to the dataset collection of on-site scanning, this study needs to fulfill at least two major aspects, as also mentioned in our previous study [56]. First, the collected point clouds should consist of Terrestrial Laser Scanning (TLS) datasets. It aims not only to accommodate more accurate 
geometric properties and better reality-based representation than Airborne Laser Scanning (ALS), but also to capture specific areas with various contextual elements, such as vegetation, temporal objects, material, and other elements, that may potentially contribute to the simulation model. Second, metadata information stored in a point cloud should at least contain typical attributes, such as $\mathrm{XYZ}$, RGB, and reflection intensity. Geometric and radiometric properties within these attributes are used to identify material properties of the existing context and to conduct environmental performance analysis.

Furthermore, to demonstrate the proposed workflow, this study collaborates with SHAU, an architectural firm located in Bandung, West Java, Indonesia, to collect 3D point cloud data to design a new public library. This project is located in Citarip, West Java, Indonesia, $\left(6^{\circ} 56^{\prime} 18.4^{\prime \prime} \mathrm{S}\right.$ $107^{\circ} 35^{\prime} 15.1^{\prime \prime} \mathrm{E}$, with ellipsoid reference WGS84) and surrounded by some vegetation and massive walls of neighboring buildings. The collected dataset was gathered using Topcon GLS-2000M incorporated with reflectors and GPS devices to achieve an accurate position during the registration process. As a light-weight and high-speed 3D laser scanner, this tool is also featured with a rugged design instrument (for use in the field) and full-dome field of view (FOV) with a selectable laser class, enabling the user to scan in extreme work environments and eye-safety concerns in dense areas. A detailed specification of the tool can be observed below (see Table 1):

Table 1. Detailed specifications of 3D scanner [71].

\begin{tabular}{cc}
\hline Parameters & Performance Specification Unit \\
\hline System performance & \\
\hline $\begin{array}{c}\text { Maximum range (at } 90 \% \text { reflectivity) } \\
\text { GLS-2000M }\end{array}$ & 350 m (standard) \\
\hline $\begin{array}{c}\text { Single point accuracy } \\
\text { Distance } \\
\text { Angle }\end{array}$ & 3.5 mm (1-150m), 1 sigma \\
$6^{\prime \prime}$
\end{tabular}

This tool's performance specifications allow the dataset collection with only three single scans to capture a sufficient scene for the selected site (see Figure 7). With these three single scans, the dataset's computational performance simulation can also be more manageable and relevant to the currently proposed workflow. Given that this approach is part of the exploratory study, architects need to 
select not only important information, but also a degree of detailed properties that are relevant to the simulation. In this regard, the setting of each scan approximately consists of four million points per scan within five minutes with a resolution $12.5 \times 12.5 \mathrm{~mm} @ 10 \mathrm{~m}$. The distance between scanners and the designated objects are principally determined based on the approximate coverage of the scanner capacity, which may cover $360^{\circ}$ of the horizontal field of view (FOV) with the distance for single-point accuracy 1-150 m. In this case, Scan 2 reaches the closest distance between the scanner and the object with $2.75 \mathrm{~m}$. This is because data scan coverage areas need to capture the surrounding wall and corner spots behind the temporary shelters. Meanwhile, the longest distance is obtained by Scan 01 with $34.9 \mathrm{~m}$, due to the diagonal position of the scanner to the corner of the site. Besides, in this dataset collection, it is worth noting that as long as the entire scenes of the selected site and relevant objects are covered, the scanner can be located at any appropriate distances.
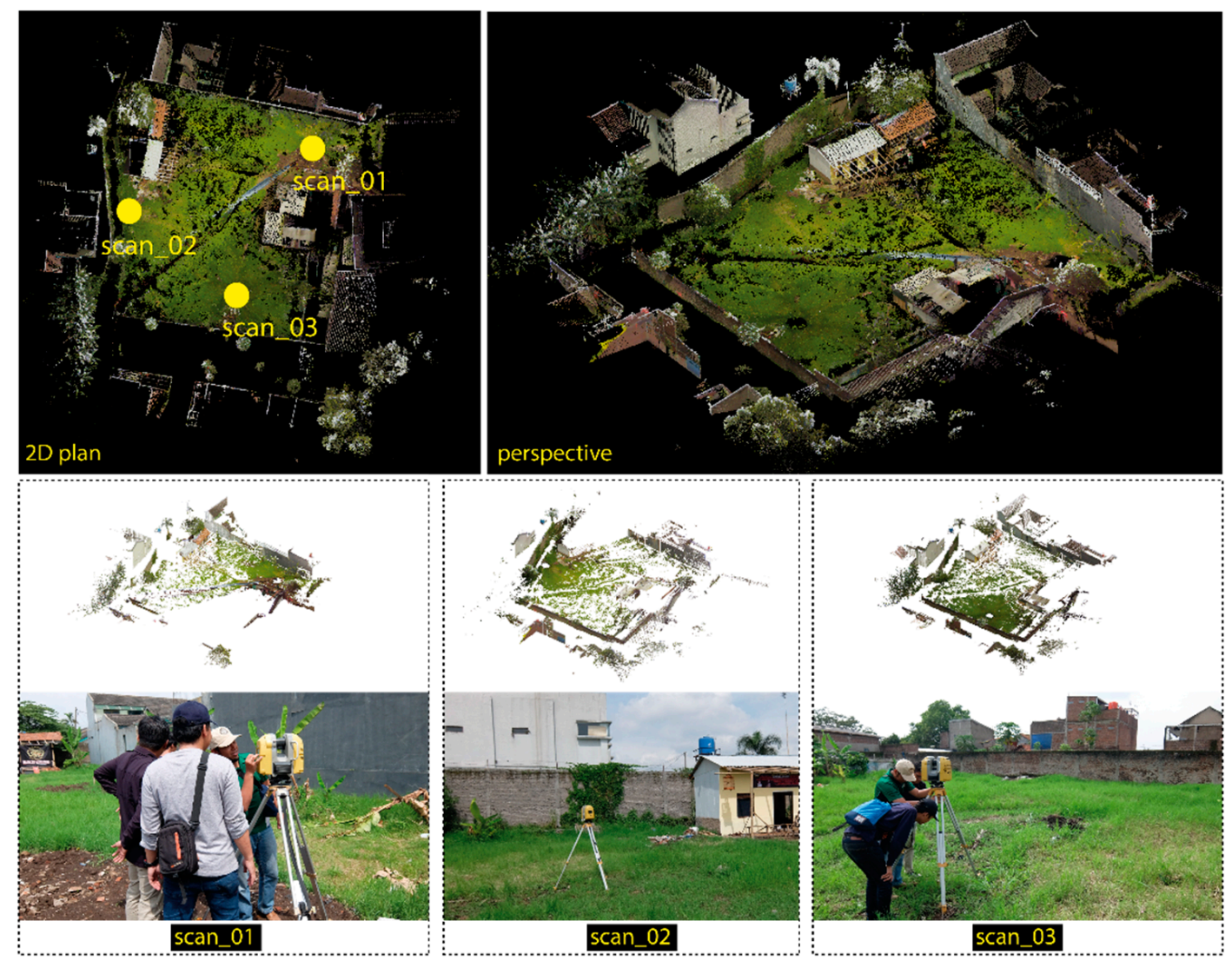

Figure 7. Dataset collection with different views captured in relation to the scanner position.

\section{Results and Discussion}

After establishing the selected dataset, this section presents the analysis results of the implemented workflow. It follows the five aforementioned steps as follows:

\subsection{Step 1-Input Parameters}

As previously described in the section about presenting the proposed workflow, input parameters consist of climatic and geographic properties. As for climatic properties, due to a constant temperature over the year in Indonesia [80], this study sets April 21st, 2019, as a sample of the selected period that represents a starting date for a dry season. Although the selected time range is limited, this simulation takes place as an exploratory study that focuses on exploring the feasibility of integrated computational workflow for shading envelopes based on attributes information of point cloud data. This specific duration is furthermore defined for four hours, starting from 11 am to $3 \mathrm{pm}$, which is averagely 
representing the highest temperature during the daylight hours. In parallel, a time-step for the simulation is set smaller than one hour to improve the simulation accuracy. This setting ultimately yielded four sun vectors that are simulated on each point of the dataset. Meanwhile, for a proposed building, the 3D polyhedra are extruded from the plot by considering predefined criteria of the buildings and local regulations, such as a $10 \mathrm{~m}$ height and 4-6 m setback requirement (see Figure 8). Each polyhedron is set to $2 \times 2 \times 2 \mathrm{~m}$ with the total number 690 units, including the generated asymmetrical shape, due to irregular order of the plot's boundary. In principle, this polyhedron's dimension can be vary depending on the architectural concept and functional program of the proposed building.

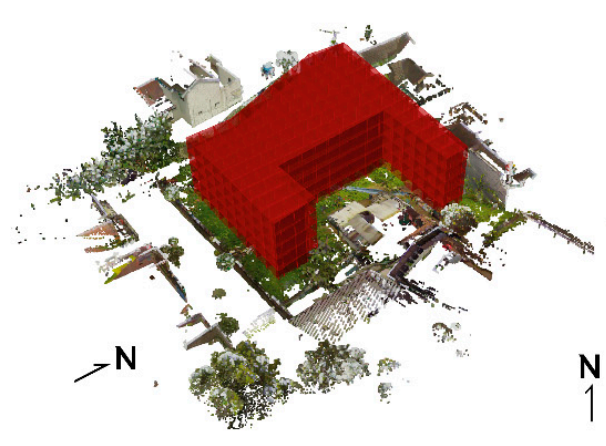

Perspective

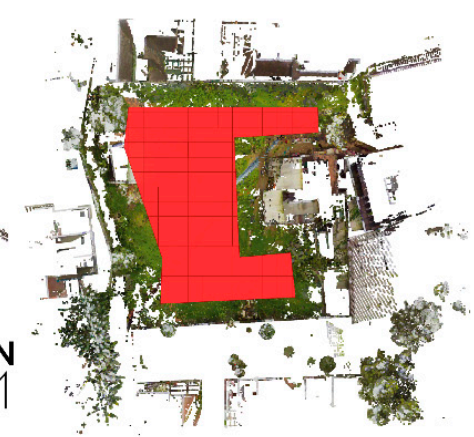

Top view

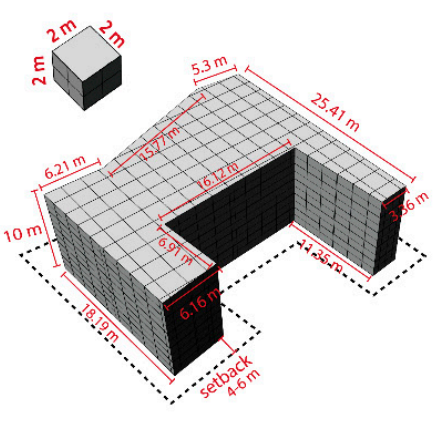

Dimension

Figure 8. Selected dataset and 3D polyhedra for a case study.

\subsection{Step 2-Dataset Preparation}

While tasks (such as sun vectors calculation and 3D polyhedra generation) have been addressed previously, this section primarily discusses the result of sequential tasks, which consist of dataset correction and dataset subsampling. The dataset correction can be performed after establishing normal values with various incident angles for each selected data scan. According to Figure 9A, all points are plotted on the basis of the cosine values of each incident angle. The pattern shown by all data scans is that points are distributed and assemble at a certain angle. This means that the laser beam projection may correspond very well at certain areas of the dataset during scanning, depending on the position of the scanner and geometric properties of the building surfaces (i.e., roughness). Afterward, to find the optimal normal values within this pattern, a standard deviation of cosine values is calculated based on a sample of points (see Figure 9B). This becomes a basis for truncating the relevant datasets based on their distribution density.

In order to determine a threshold value of the dataset truncation, a standard deviation of the whole population of the dataset is plotted (see Figure 9C). This facilitates us to generate the cosine values pattern resulting in Figure 9B so that the number of points with a high distribution level of density can be identified reliably. As a result, ground areas of the plot (refer to blue-coded pattern in Figure 9B) that mostly contain grass are automatically removed, due to irrelevant properties with the predefined criteria, while datasets with a red pattern are used for the intensity correction (See Figure 10). 


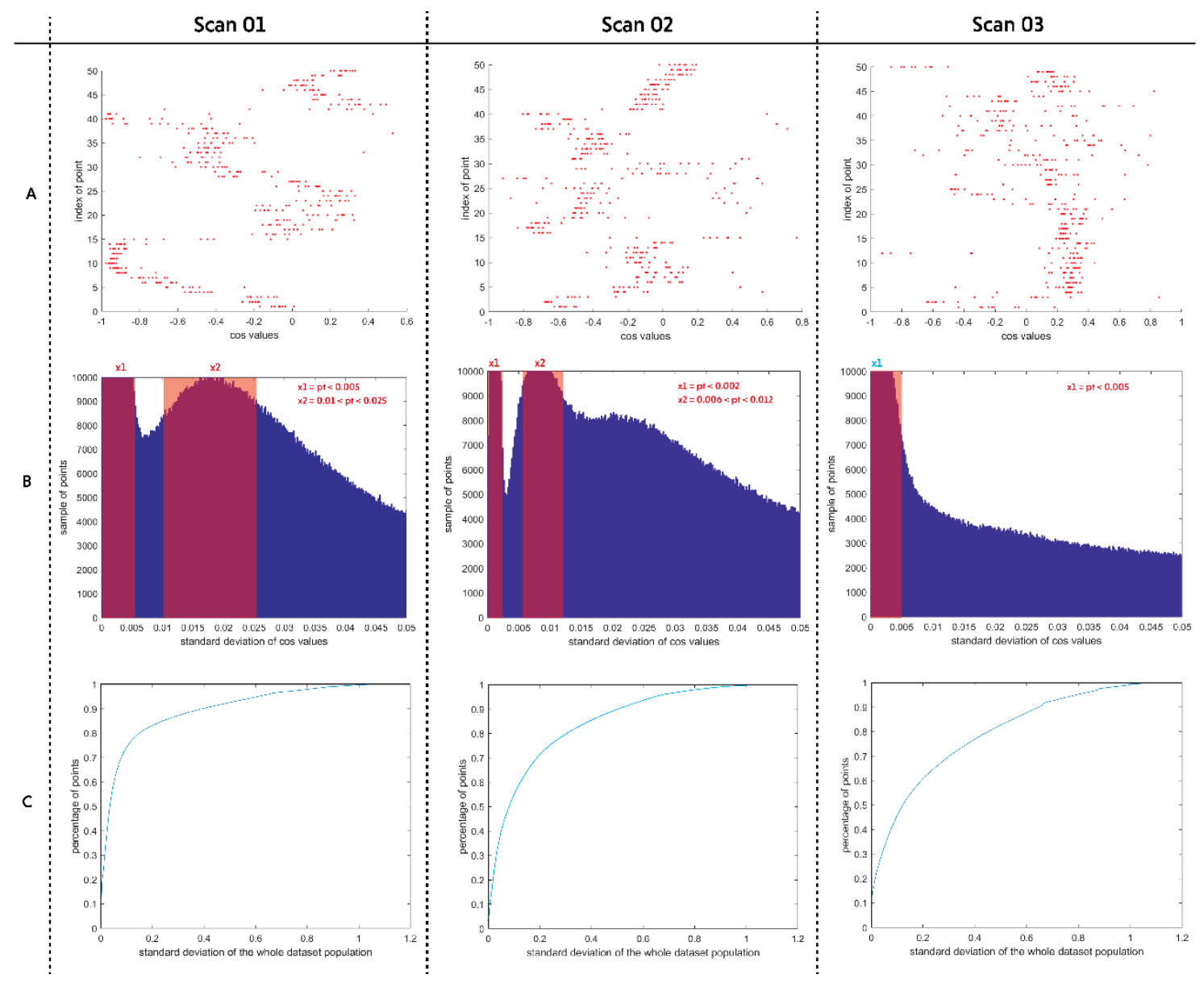

Figure 9. Dataset correction (A). Point distributions based on cosine values of each incident angle (B). The standard deviation of cosine values based on a group sample of points $(\mathbf{C})$. The standard deviation of the whole dataset population.

Figure 10 specifically demonstrates the result of intensity correction from the raw attributes to the corrected one through the scalar field of intensity and intensity distribution values. The dataset transformations of each scan are plotted based on four-color steps, ranging from blue to yellow, which represent the lowest and the highest intensity values. The threshold between these values is represented by light and dark green. The trend clearly shows that the raw intensity of all data scans contains yellow areas (refers to the "before" part), representing high reflective surfaces. However, these areas predominantly assign incorrect surface materials as it should be. For example, some parts of the buildings, such as wood façade and clay roof tiles, are attributed to yellow intensity. In fact, these materials principally contain a high level of emissivity value or low intensity, which means that the return incident energy from these materials to the scanner is highly decreased, due to its spectral reflectance mechanism on rough, dark, and dull surfaces [81]. In addition, the intensity correction also compensates for scanned areas around the scanner, due to the impact of the brightness level of intensity. These areas are massively indicated with yellow-coded values, due to the high level of intensity produced by the scanner at a very short distance. In this case, the scanner's brightness reducer is assumed not entirely and comprehensively applied in these areas, especially within the distance range of $10 \mathrm{~m}$ from the scanner position. This is because some local constraints, such as atmospheric variables (i.e., humidity and temperature pressures) and surface roughness of the objects, may influence specular and diffuse reflection of the laser pulse during scanning [54]. 


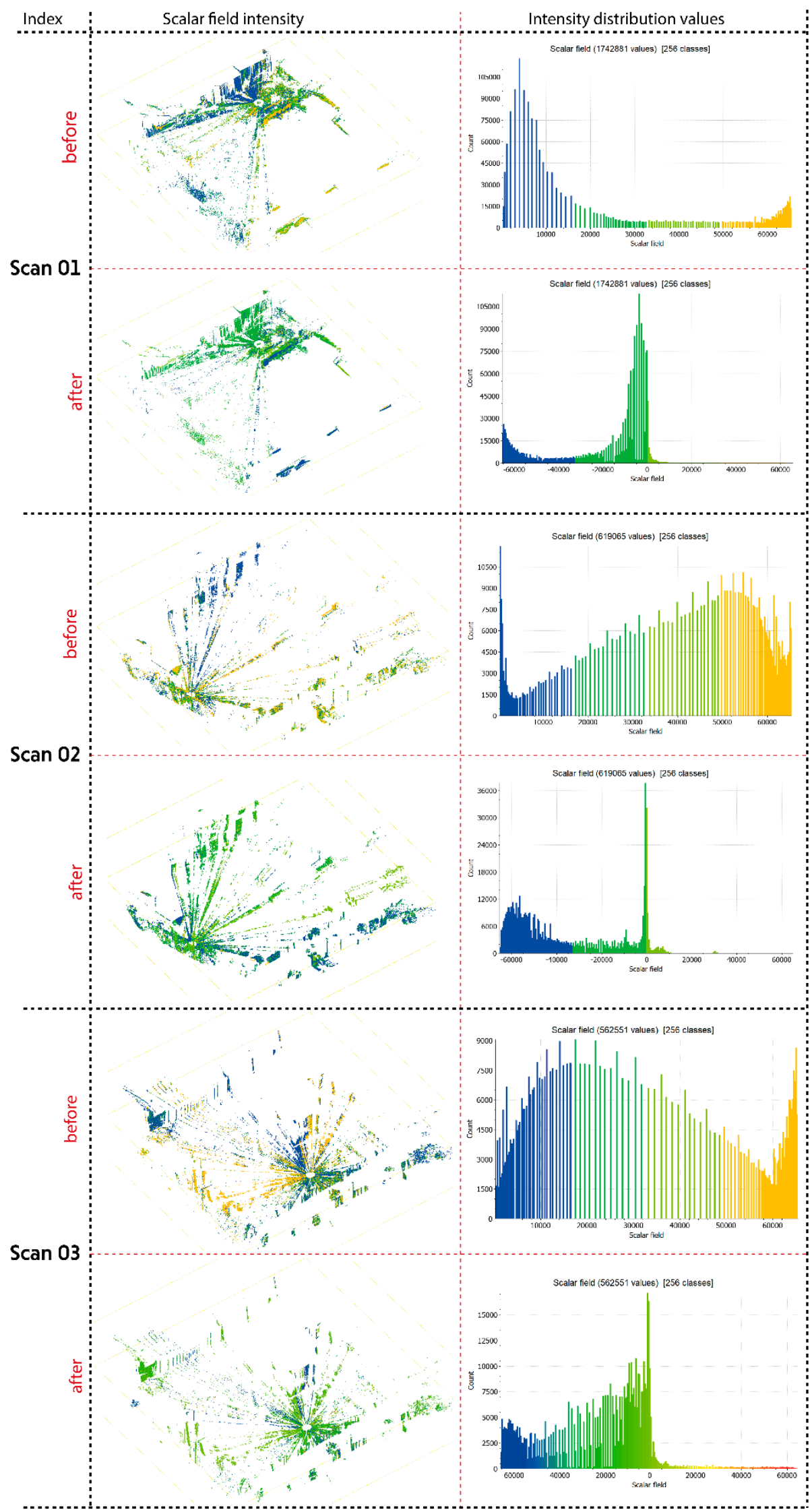

colour codes

Figure 10. Intensity correction on each data scan. 
The corrected datasets are then performed with the subsampling procedure in CC in order to control the unstructured point density when it comes to the simulation. In this case, the distance between points is set to 0.05 or equal to $5 \mathrm{~cm}$, which results, on average, in a decrease point, up to $70 \%$ of the truncated datasets. This procedure, however, only works on position information (XYZ) because it can cause interoperability issues when it transforms into the 3D model in Rhino, due to the different nature of the algorithmic operation. To tackle this issue, index information of each point (refers to ID in Figure 11) needs to be extracted beforehand during the truncation process and then used to synchronize the original attributes (i.e., XYZ and RGB) with the corrected ones in the 3D model. The workflow of this dataset transformation is illustrated further in Figure 11.

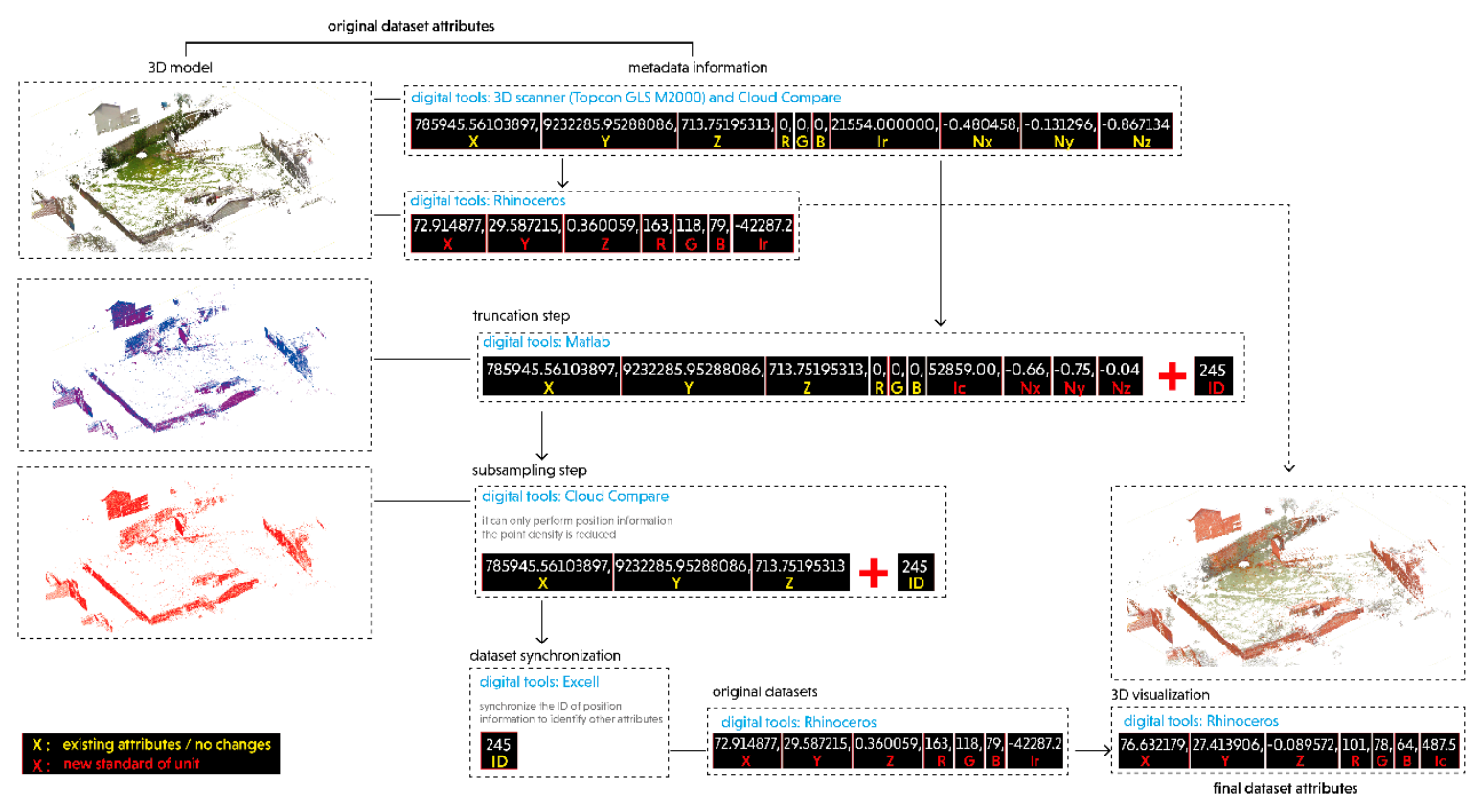

Figure 11. Transformation of the dataset attributes.

In general, Figure 11 demonstrates a unit conversion of the dataset attributes that take place according to the step and digital tool used, starting from the raw data scan to the final stage of the workflow. A major transformation principally occurs when converting the original datasets from the 3D scanner to the 3D model in Rhino, which changes the unit of position (XYZ), color (RGB), and the raw intensity values (Ir). Therefore, the interoperability aspect plays a critical role not only to support the simulation analysis on the metadata information of the dataset (i.e., correction, truncation, and subsampling step), but also to help the visualization of geometric configuration based on the selected attributes.

\subsection{Step C-Selection Criteria}

Before running the simulation, the subsampled datasets are evaluated based on the criteria of sun visibility and albedo values. These criteria identify relevant points that will be used for the voxels generation of shading envelopes. According to Figure 12, the resulting points for both criteria are significantly decreased as compared to the total points from the subsampled datasets. This is because a majority of subsampling points on the ground areas contain zero and negative sun vector values, which do not fulfill the sun visibility criteria. Accordingly, these points are automatically isolated, while successful ones are used as the basis datasets to perform the criteria of albedo values and the ray-tracing analysis.

In general, the trend in Figure 12 shows that the majority of selected points both for albedo values and the visible sun are similar and overlapping in a certain spot, such as the surrounding areas of the scanner position. On the other hand, each data scan also illustrates different specific 
areas for point distribution. For example, points for Scan 01 are primarily detected on the edge of the building's roof, while Scan 02 and Scan 03 are partially distributed in the brick wall and clay roofs of the surrounding houses, respectively. Furthermore, the selected datasets for each data scan are then used in the simulation process. This will be presented in the following step.

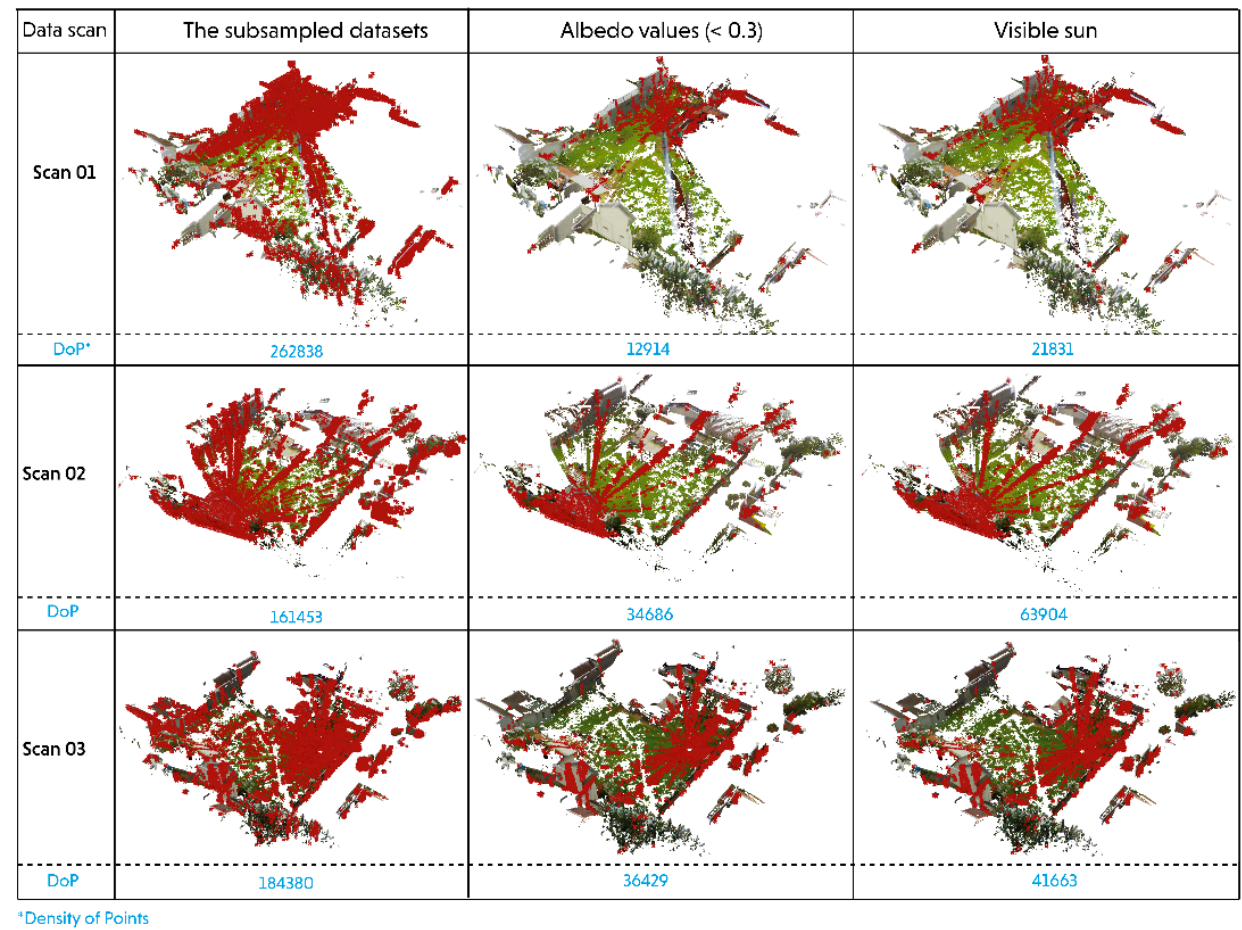

Figure 12. The resulting points after performing the selection criteria.

\subsection{Step D-Form Generation Process of Shading Envelopes}

The resulting datasets that successfully fulfill the predefined criteria in the previous step are used in the simulation by following a series of tasks illustrated in Figure 5. The outputs of these tasks are demonstrated in Figure 13. It consists of five steps to generate volumetric shapes and three steps to simulate the ray-tracing analysis. Beforehand, each data scan is divided into two parts in order to minimize the high computational cost during the simulation. According to Figure 13, some analysis can be further discussed as follows:

- The ray-tracing analysis (see part RTA-01) shows the intersection lines that occur between visible sun vectors, selected points of the existing site, and 3D polyhedra. The result of these intersections is then illustrated in voxels-01, which indicate a group of voxels that fulfill the criteria of receiving direct sun access. In this case, the simulation of Scan 03 yields a lower number of voxels-01 as compared with other scans, due to the density and position of each point to the 3D polyhedra. Specifically, the existing datasets of Scan 03 approximately cover all sides of the 3D polyhedra during the simulation, while Scan 01 only intersects a certain part of the polyhedra because the existing points are predominantly distributed on one side. Conversely, the resulting voxels-02 show a contrasting number of voxels, due to the different sun access criteria.

- Voxels-03 illustrate a simulation result of shading criteria. A major trend is shown by Scan 02, which results in a significantly decreasing number of voxels from the voxels-02. This is because sun vectors originated from the reference points of voxels- 02 are massively intersected with the geometric shape of voxels-01.

- Voxels-04 represent a group of polyhedra that block the direct sun access to certain areas of surrounding properties. Each data scan consists of a different voxel configuration depending 
on the selected areas that have been identified below the albedo values 0.3 . On the other hand, voxels- 05 constitute as concatenating polyhedra gathered from the resulting voxels-02, voxels- 03 , and voxels-04. Although the volumetric size of voxels-05 shows a significant improvement, especially for Scan 03, unfortunately, the resulting voxels can only compensate for the shading condition of the surrounding properties and still lack in protecting the proposed building from the direct sun access within a certain angle. Accordingly, a self-shading envelope workflow needs to be applied afterwards.

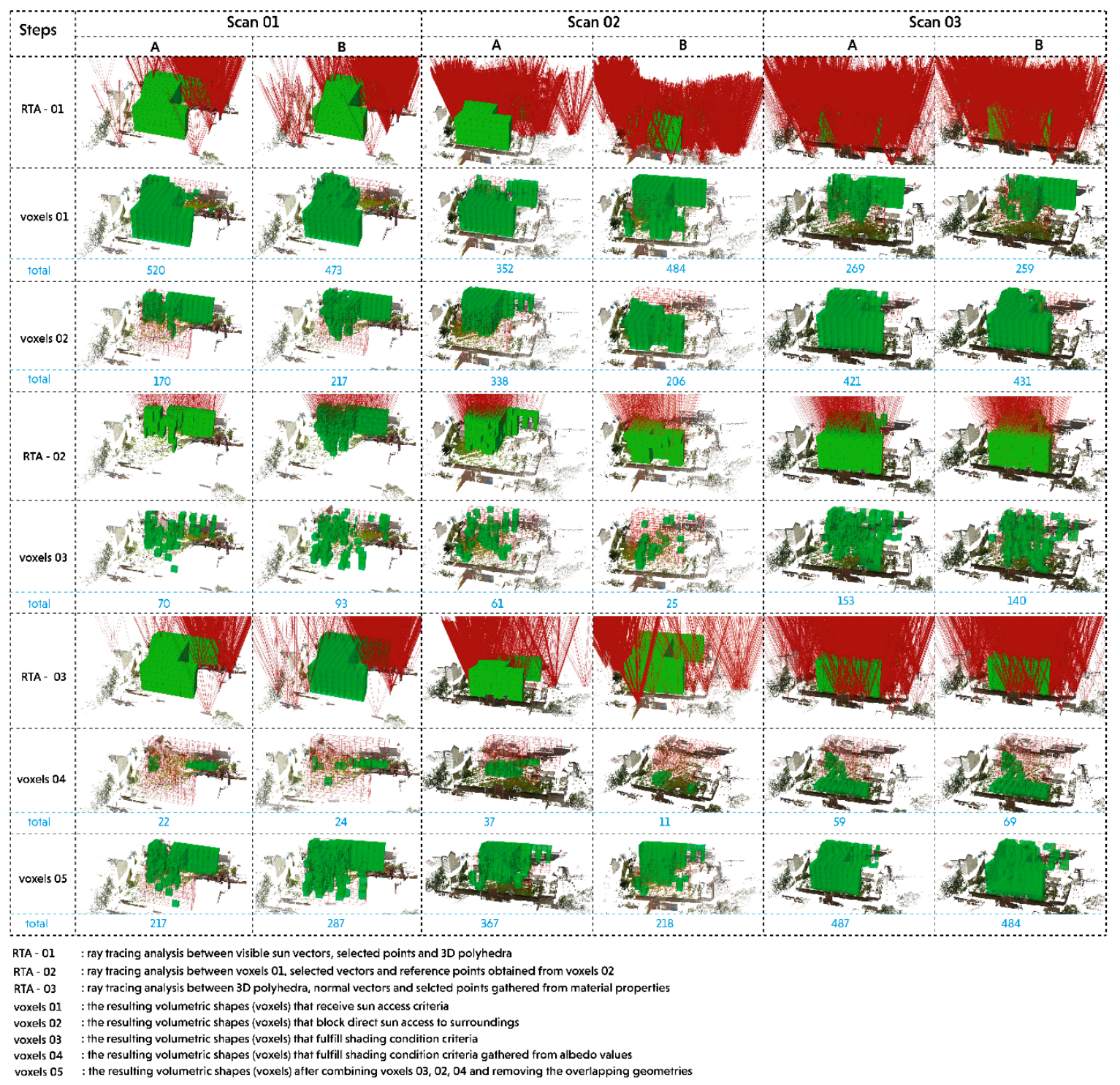

Figure 13. The geometric configuration of subtractive shading envelopes based on sequential steps of design simulation.

\subsection{Step E-Form Generation Process of Self-Shading Envelopes}

In order to perform self-shading envelopes, solar protection planes are applied on the voxels-05 by excluding voxels located on the upper part of the 3D polyhedra. This is because the upper voxels are considered as the roof or shelter for the remaining voxels located on the bottom envelopes. To do so, the upper voxels in each data scan are firstly separated from the set of 3D polyhedra (see Figure 14 step 02), while performing the ray-tracing analysis (see step 03) on the remaining voxels. As a result (see steps 04), voxels-06 illustrate the resulting envelopes that fulfill the criteria of a self-shading 
mechanism. The trend shows that the resulting voxels- 06 for Scan 03 reduce, on average, $50 \%$ of the volumetric 3D polyhedra (refers to voxels-05 bottom part), while other data scans also decrease nearly a half of the initial 3D polyhedra. In this case, most of the reducing voxels are located on the edge of the 3D polyhedra or voxels that act as an exterior wall of the $3 \mathrm{D}$ polyhedra.

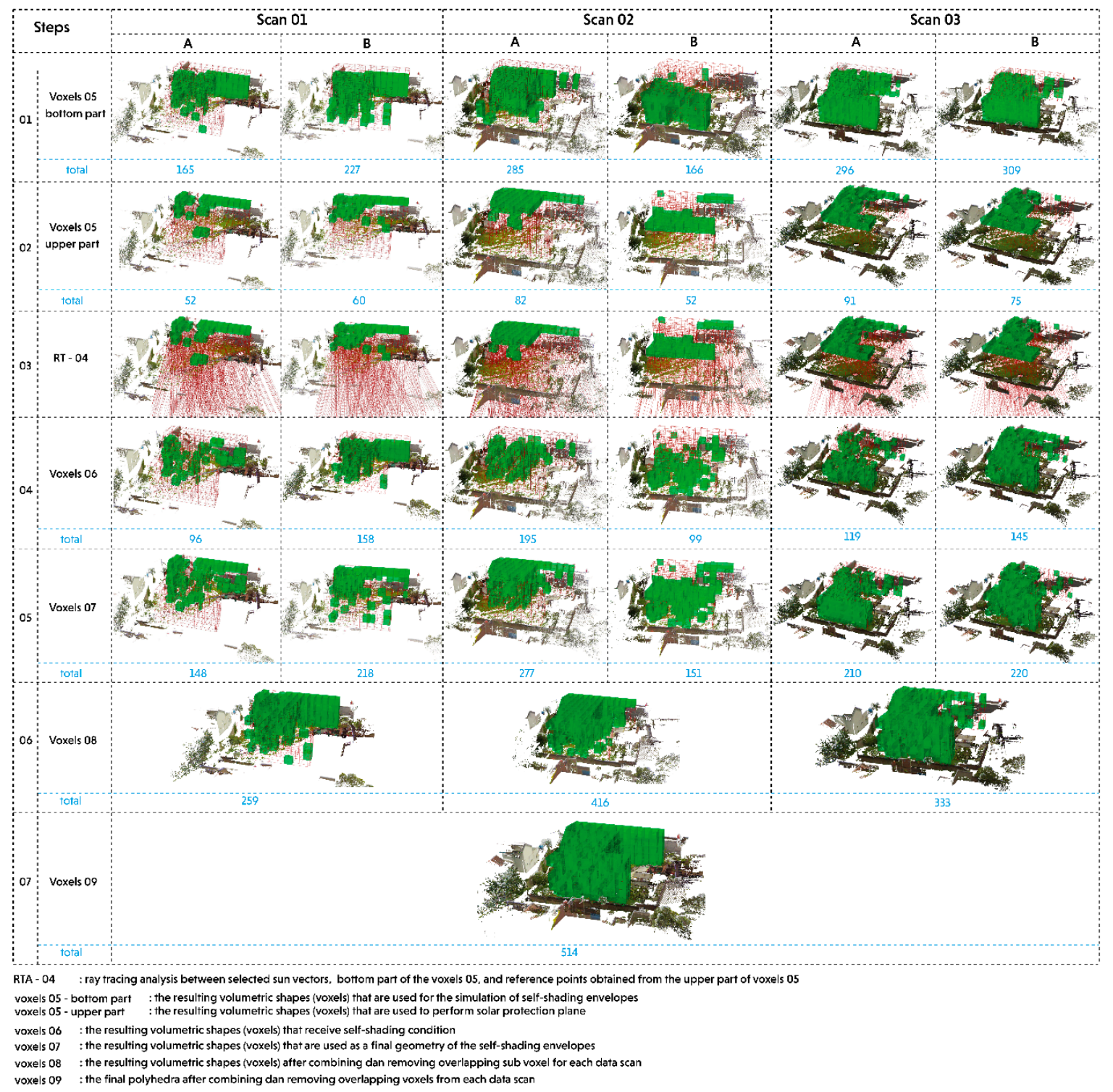

Figure 14. Geometric configuration of subtractive shading envelopes.

Voxels-06 of each data scan is combined with the upper part voxels that are previously separated in the previous step (refers to voxels-05 step 02). This process results in voxels-07 (refers to step 05), which are then used to perform the merging procedure in step 06 -voxels- 08 . This step also includes elimination procedures for the voxels located in the same location. In so doing, the total number of polyhedra represented by voxels- 08 shows the core geometry for each data scan. For example, the initial amount of voxels- 08 after combining Scan $1 \mathrm{~A}$ and $1 \mathrm{~B}$ is 366 polyhedron, but after eliminating the overlapping polyhedron, it yields 259 polyhedra. Thus, 107 voxels are indicated as overlaps during the merging procedure. Afterward, the voxels-08 of each data scan are merged using the same steps in order to generate the final geometry of self-shading envelopes. Ultimately, the total number of voxels that fulfill the shading criteria for surrounding contexts and the 3D polyhedra is 514 of 690 (see Figure 14). 
Furthermore, the resulting geometries of self-shading envelopes can be identified as a core geometry that fulfills the building's main activities based on the daylight condition. For example, architects may plot voxels located on the ground level as a communal space, workshops, and playing areas for children as it requires more open activities while the upper floor can be fulfilled with a reading room or any other activities that match with the required shading. In addition, the roof of this upper floor can be utilized as a solar collector for PV panels that may produce supplementary solar energy for the buildings. This is relevant because Indonesia's geographical condition is located in the Equator can support the electricity production around 1,534 $\mathrm{kWh} /$ year for each installed solar panel based on $4.5 \mathrm{kWh} / \mathrm{m}^{2}$ average daily radiation [82]. As compared to the existing method of self-shading envelopes proposed by Capeluto [36], this approach not only creates potential shading that merely comes from the roof of the envelopes, but also originates from a dynamic form of the envelope facades that correspond to different solar vectors. For these reasons, geometrical configurations of the final envelopes in this study show more variation in different orientations.

In design practices, the proposed approach can be adopted by architects as a further step for designing and analyzing high performing envelopes both for new and existing development areas. The Dutch architecture and urban design firm, MVRDV, has been implemented the basic design principles of this approach into several projects based on solar-oriented designs. As a new development area, for example, the P15 Ravel Plot, which is located in the Zuidas district, 1082 LC, Amsterdam was constructed based on the idea of the optimal line of sight integrated with the three-dimensional landscape and greenery [83]. Meanwhile, in urban scale, MVRDV proposed the idea of solar energy as a part of design intervention for zero energy neighborhoods to the existing historic infrastructures in Bordeaux, France [84]. These examples clearly show a stepping-stone for architectural design practices to explore further the relevance and potential application of a voxel-based design approach in supporting sustainable environmental design. In this regard, point cloud data can be a powerful instrument that fortifies environmental performance simulation of design context during the early design phase.

\section{Conclusions}

This study investigates the potential application of attribute information stored in point cloud data to support a new computational method for a voxel-based design approach based on shading performance criteria. As a part of the contextual design strategy, the proposed workflow specifically presents form generation based on subtractive shading envelopes and material properties of existing contexts that are used to generate a new method for self-shading envelopes in tropical countries. Ultimately, this integrated approach may support architects in taking a comprehensive design decision during the early stage of the design process based on real contextual datasets. As an exploratory study, this work presents several concluding remarks as follows:

- The attribute information of point cloud data (i.e., $X Y Z, R G B$, and reflection intensity) contributes not only to calculate material properties of existing context, but also to be a part of selection criteria for generating voxel-based subtractive shading envelopes.

- The dataset preparation includes pre-processing and correction steps that help architects minimize the environmental effects of the dataset measurement during scanning and to select reliable and relevant information for the contextual analysis and the simulation process.

- The proposed workflow enables architects to produce more variation of geometrical facades, especially related to the final geometry of self-shading envelopes that are not only depending on the roof perimeters, but also considering reference points that attach to the upper part of each generated voxel.

- The ray-tracing analysis between 3D polyhedra and selected points of surrounding contexts permits one to identify specific areas and voxels that fulfill shading performance criteria during a predefined period. 
- As a contextual design approach, self-shading envelopes not only receive environmental performance responses from surrounding buildings to the proposed design, but also deliver feedback from the new building to the existing contexts.

Despite the findings mentioned above, there still some limitations. For example, first, the implementation of this proposed workflow in design practice may require a collaboration with the field of remote sensing, especially related to the dataset collection and preparation. This is because some specific tasks need prior knowledge regarding the dataset pre-processing and particular digital tools. Second, the limitations of simulating highly dense datasets imply the number of solar vectors that need to be reduced, especially when dealing with the ray-tracing analysis procedure. Third, normal surface of the datasets and voxels of the proposed building should be evaluated by each solar vector, but as a consequence, it requires high computational processing that is currently lacking in our 3D modeling tools. Therefore, further research is expected to consider these issues in order to enhance the quality of the simulation results. It is also recommended to implement this study in different urban settings with multiple building functions, such as high-rise or new development areas, so that various urban forms can be further explored.

Author Contributions: Conceptualization, F.D.L. and M.F.A.; methodology, M.T. and M.F.A.; writing一original draft preparation, M.F.A.; writing—review and editing, M.T. and F.D.L.; visualization, M.F.A.; supervision, S.S.; All authors have read and agreed to the published version of the manuscript.

Funding: This research was funded by Indonesia Endowment Fund for Education (LPDP) as a part of PhD Research of the first author under the chair of Design Informatics, Faculty of Architecture and the Built Environment of TU Delft. In addition, the European Regional Development Fund grant (the Estonian Centre of Excellence in Zero Energy Resource Efficient Smart Buildings and Districts) ZEBE n. 2014-2020.4.01.15-0016 and the European Commission H2020 grant Finest Twins n. 856602 are used to support the work of the second author.

Acknowledgments: We thank Indonesian Endowment Fund for Education (LPDP) for funding a PhD research of the first author under the chair of Design Informatics, Faculty of Architecture and the Built Environment of TU Delft, the European Regional Development Fund grant (the Estonian Centre of Excellence in Zero Energy Resource Efficient Smart Buildings and Districts) ZEBE n. 2014-2020.4.01.15-0016 and the European Commission H2020 grant Finest Twins n. 856602 for supporting the work of the second author. In addition, the authors would like to express their gratitude to SHAU in Bandung for providing access to their projects, Irwan Gumilar from the study program of Geodesy and Geomatics Engineering, ITB and Haidar from PT Asaba for assisting us in collecting the dataset, Yu-Chou Chiang for his technical support during dataset processing, James Nelson in TU Delft urbanism editing team for proofreading the preliminary manuscript, and TU Delft Library for supporting logistics and open access of this journal publication.

Conflicts of Interest: The authors declare no conflict of interest.

\section{References}

1. Shih, N.-J.; Wu, M.-C. A 3D Point-Cloud-Based Verification of as-Built Construction Progress. In Proceedings of the CAAD Futures 2005 Conference, Vienna, Austria, 20-22 June 2005.

2. Alsadik, B.; Gerke, M.; Vosselman, G. Visibility analysis of point cloud in close range photogrammetry. ISPRS Ann. Photogr. Remote Sens. Spat. Inf. Sci. 2014, 2, 9-16. [CrossRef]

3. Andriasyan, M.; Moyano, J.J.; Julián, J.E.N.; García, D.A. From Point Cloud Data to Building Information Modelling: An Automatic Parametric Workflow for Heritage. Remote Sens. 2020, 12, 1094. [CrossRef]

4. Shanoer, M.M.; Abed, F.M. Evaluate 3D laser point clouds registration for cultural heritage documentation. Egypt. J. Remote Sens. Space Sci. 2018, 21, 295-304. [CrossRef]

5. Remondino, F. Heritage Recording and 3D Modeling with Photogrammetry and 3D Scanning. Remote Sens. 2011, 3, 1104-1138. [CrossRef]

6. Núñez-Andrés, M.A.; Buill, F.; Costa-Jover, A.; Puche, J.M. Structural assessment of the Roman wall and vaults of the cloister of Tarragona Cathedral. J. Build. Eng. 2017, 13, 77-86. [CrossRef]

7. Bornaz, L.; Lingua, A.; Rinaudo, F. Engineering and Enviroonmental Applications of Laser Scanner Techniques. In Proceedings of the ISPRS Commission III Symposium Photogrammetric Computer Vision, Graz, Austria, 9-13 September 2002.

8. Kassner, R.; Koppe, W.; Schüttenberg, T.; Bareth, G. Analysis of the Solar Potential of Roofs By Using Official Lidar Data. In Proceedings of the ISPRS Congress, Beijing, China, 3-11 July 2008. 
9. Carneiro, C.; Morello, E.; Desthieux, G. Assessment of Solar Irradiance on the Urban Fabric for the Production of Renewable Energy using LIDAR Data and Image Processing Techniques. In Proceedings of the Advances in GIScience, Hannover, Germany, 2-5 June 2009.

10. Desthieux, G.; Carneiro, C.; Camponovo, R.; Ineichen, P.; Morello, E.; Boulmier, A.; Abdennadher, N.; Dervey, S.; Ellert, C. Solar Energy Potential Assessment on Rooftops and Facades in Large Built Environments Based on LiDAR Data, Image Processing, and Cloud Computing. Methodological Background, Application, and Validation in Geneva (Solar Cadaster). Front. Built Environ. 2018, 4, 1-22. [CrossRef]

11. Staneva, N.N. Approaches for generating 3D solid models in AutoCAD and solid works. J. Eng. 2008, 3, 28-31.

12. Shapiro, V. Spatial Automation Laboratoty. In Solid Modeling; University of Wisconsin: Madison, WI, USA, 2001.

13. Otepka, J.; Ghuffar, S.; Waldhauser, C.; Hochreiter, R.; Pfeifer, N. Georeferenced Point Clouds: A Survey of Features and Point Cloud Management. ISPRS Int. J. Geoinf. 2013, 2, 1038-1065. [CrossRef]

14. Richter, R.; Döllner, J. Concepts and techniques for integration, analysis and visualization of massive 3D point clouds. Comput. Environ. Urban Syst. 2014, 45, 114-124. [CrossRef]

15. Arayici, Y. Towards building information modelling for existing structures. Struct. Surv. 2008, 26, $210-222$. [CrossRef]

16. Alkadri, M.F.; Turrin, M.; Sariyildiz, S. The use and potential applications of point clouds in simulation of solar radiation for solar access in urban contexts. Adv. Comput. Des. 2018, 3, 319-338.

17. Knowles, R.L. Energy and Form: An Ecological Approach to Urban Growth, Cambridge; The MIT Press: Cambridge, MA, USA, 1974.

18. Knowles, R.L. Sun, Rhytm and Form, Massachusetts; The MIT Press: Cambridge, MA, USA, 1981.

19. Alkadri, M.F.; De Luca, F.; Turrin, M.; Sariyildiz, S. Understanding Computational Methods for Solar Envelopes Based on Design Parameters, Tools, and Case Studies: A Review. Energies 2020, 13, 3302. [CrossRef]

20. GlobalABC, IEA and UN. 2019 Global Status Report for Buildings and Construction: Towards a Zero-Emission, Efficient and Resilient Buildings and Construction Sector; IEA and United Nations Environment Programme: Madrin, Spain, 2019.

21. Chandel, S.; Sharma, V.; Marwah, B.M. Review of energy efficient features in vernacular architecture for improving indoor thermal comfort conditions. Renew. Sustain. Energy Rev. 2016, 65, 459-477. [CrossRef]

22. Ehzan, M.; Farshchi, M.A.; Ford, A. Vernacular Architecture And Energy Use In Buildings: A Comparative Study. IJAMCE 2015, 2, 35-42.

23. Lepore, M. The right to the sun in the urban design. Vitr. Int. J. Arch. Technol. Sustain. 2017, 2, $24-43$. [CrossRef]

24. Mazzone, D. The Dark Side of a Model Community: The Ghetto of el-Lahun. J. Anc. Egypt. Archit. 2017, 2, 19-54.

25. Butti, K.; Perlin, J. A Golden Threat 2500 Years of Solar Architecture and Technology; Van Nostrand Reinhold Company: New York, NY, USA, 1980.

26. Giacomo, L. The Architecture of A. Palladio in Four Books; John sWatts: London, UK, 1715.

27. Curreli, S.; Coch, H. Solar access in the compact city: A study case in Barcelona. In Proceedings of the 3rd International Conference PALENC 2010 Passive \& Low Energy Cooling for the Built Environment, Rhodes Island, Greece, 29 September-1 October 2010.

28. Wang, X.; Wei, Z.-S.; Pang, X. Solar design in the application of the city planning. In Proceedings of the International Conference on Low-Carbon Transportation and Logistics, and Green Buildings, Beijing, China, 12-13 October 2012.

29. Sarkar, A. Low energy urban block: Morphology and urban guidelines. In Proceedings of the 45th ISOCARP Congress, Porto, Portugal, 18-22 October 2009.

30. Littlefair, P.J.; Santamouris, M.; Alvarez, S.; Dupagne, A.; Hall, D. Environmental Site Layout Planning: Solar Access, Microclimate and Passive Cooling in Urban Areas; Construction Research Communications Ltd.: London, $\mathrm{UK}, 2000$.

31. Dekay, M.; Brown, G.Z. Sun, Wind \& Light: Architectural Design Strategies, 3th ed.; John Wiley \& Sons: Hoboken, NJ, USA, 2014. 
32. Emmanuel, R. A Hypothetical 'Shadow Umbrella' for Thermal Comfort Enhancement in the Equatorial Urban Outdoors. Arch. Sci. Rev. 1993, 36, 173-184. [CrossRef]

33. DeKay, R.M. Climatic urban design: Configuring the urban fabric to support daylighting, passive cooling, and solar heating. Sustain. City VII 2012, 1, 619-630.

34. Dinić, M.; Mitković, P. Planning regulations in the USA and their implications on urban design in the central city zone. Facta Univ. Arch. Civ. Eng. 2011, 9, 289-299. [CrossRef]

35. Szokolay, S.V. Introduction to Architectural Science: The Basis of Sustainable Design; Architectural Press: Oxford, UK, 2004.

36. Capeluto, I.G. Energy performance of the self-shading building envelope. Energy Build. 2003, 35, $327-336$. [CrossRef]

37. Yezioro, A.; Shaviv, E. Shading: A design tool for analyzing mutual shading between buildings. Sol. Energy 1994, 52, 27-37. [CrossRef]

38. Marsh, A. Computer-Optimised Shading Design. In Proceedings of the Eight International IBPSA Conference, Eindhoven, The Netherlands, 11-14 August 2003.

39. Stevanović, S.; Stevanović, D.; Dehmer, M. On optimal and near-optimal shapes of external shading of windows in apartment buildings. PLoS ONE 2019, 14, e0212710. [CrossRef] [PubMed]

40. Kabre, C. Winshade: A computer design tool for solar control. Build. Environ. 1998, 34, 263-274. [CrossRef]

41. Etzion, Y. An improved solar shading design tool. Build. Environ. 1992, 27, 297-303. [CrossRef]

42. Sargent, J.; Niemasz, J.; Reinhart, C.F. In Proceedings of the SHADERADE: Combining Rhinoceros and Energyplus for the Design of Static Exterior Shading Devices, Sydney, Australia, 14-16 November 2011.

43. Kaftan, E.; Marsh, A. Integrating the cellular method for shading design with a thermal simulation. In Proceedings of the International Conference "Passive and Low Energy Cooling for the Built Environment", Santorini, Greek, 19-21 May 2005.

44. Leidi, M.; Schlüter, A. Exploring urban space-Volumetric site analysis for conceptual design in the urban context. Internatl. J. Archit. Comput. 2013, 11, 157-182.

45. Leidi, M.; Schlüter, A. Volumetric insolation analysis. In Proceedings of the CleanTech for Sustainable Buildings-From Nano to Urban Scale (CISBAT 2011), Lausanne, Switzerland, 14-16 September 2011.

46. Ozel, F. SolarPierce: A Solar Path-Based Generative System. In Proceedings of the Computation and Performance-Proceedings of the 31st eCAADe Conference, Delft, The Netherlands, 18-20 September 2013.

47. Da Veiga, J.; La Roche, P. A Computer Solar Analysis Tool for the Design and Manufacturing of Complex Architectural Envelopes: EvSurf. In Proceedings of the SIGraDi 2002, 6th Iberoamerican Congress of Digital Graphics, Caracas, Venezuela, 27-29 November 2002.

48. Littlefair, P. Passive solar urban design: Ensuring the penetration of solar energy into the city. Renew. Sustain. Energy Rev. 1998, 2, 303-326. [CrossRef]

49. De Luca, F. Solar form finding. In Proceedings of the 37th Annual Conference of the Association for Computer Aided Design in Architecture: Disciplines and Disruption, ACADIA, Cambridge, UK, 2-4 November 2017.

50. De Luca, F.; Dogan, T. A novel solar envelope method based on solar ordinances for urban planning. Build. Simul. 2019, 12, 817-834. [CrossRef]

51. De Luca, F.; Voll, H. Computational method for variable objectives and context-aware solar envelopes generation. In Proceedings of the 8th Annual Symposium on Simulation for Architecture and Urban Design, SimAUD, Toronto, ON, Canada, 22-24 May 2017.

52. Darmon, I. Voxel computational morphogenesis in urban context: Proposition and analysis of rules-based generative algorithms considering solar access. In Proceedings of the Advanced Building Skins, Bern, Switzerland, 28-29 October 2019.

53. Kaasalainen, S.; Krooks, A.; Kukko, A.; Kaartinen, H. Radiometric Calibration of Terrestrial Laser Scanners with External Reference Targets. Remote Sens. 2009, 1, 144-158. [CrossRef]

54. Kashani, A.G.; Olsen, M.J.; Parrish, C.E.; Wilson, N. A Review of LIDAR Radiometric Processing: From Ad Hoc Intensity Correction to Rigorous Radiometric Calibration. Sensors 2015, 15, 28099-28128. [CrossRef]

55. Alkadri, M.F.; De Luca, F.; Turrin, M.; Sariyildiz, S. Making use of point cloud for generating subtractive solar envelopes. In Proceedings of the eCAADe SIGraDi 2019: Architecture in the Age of the 4th Industrial Revolution, Porto, Portugal, 7 September 2020. 
56. Alkadri, M.; De Luca, F.; Turrin, M.; Sariyildiz, I.S. An integrated approach to subtractive solar envelopes based on attribute information from point cloud data. Renew. Sustain. Energy Rev. 2020, 123, 109742. [CrossRef]

57. Fujita, Y.; Hoshino, Y.; Ogata, S.; Kobayashi, I. Attribute Assignment to Point Cloud Data and Its Usage. Glob. J. Comp. Sci. Technol. 2015, 15, 2.

58. Zhan, Q.; Yu, L.; Liang, Y. A point cloud segmentation method based on vector estimation and color clustering. In Proceedings of the 2nd International Conference on Information Science and Engineering (ICISE), Hangzhou, China, 4-6 December 2010.

59. Kobayashi, I.; Fujita, Y.; Sugihara, H.; Yamamoto, K. Attribute Analysis of Point Cloud Data with Color Information. J. Jpn. Soc. Civ. Eng. Ser. F3 Civil Eng. Inform. 2011, 67. [CrossRef]

60. Aijazi, A.K.; Checchin, P.; Trassoudaine, L. Segmentation Based Classification of 3D Urban Point Clouds: A Super-Voxel Based Approach with Evaluation. Remote Sens. 2013, 5, 1624-1650. [CrossRef]

61. Fujita, Y.; Kobayashi, I.; Chanseawrassamee, W.; Hoshino, Y. Application of Attributed Road Surface Point Cloud Data in Road Maintenance. J. Jpn. Soc. Civ. Eng. Ser. F3 Civil Eng. Inform. 2014, 70. [CrossRef]

62. Tamke, M.; Blümel, I.; Ochman, S.; Vock, R.; Wessel, R. From point clouds to definitions of architectural space: Potentials of automated extraction of semantic information frompoint clouds for the building profession. In Proceedings of the eCAADe, Newcastle upon Tyne, UK, 10 September 2014.

63. Turner, E.; Cheng, P.; Zakhor, A. Fast, Automated, Scalable Generation of Textured 3D Models of Indoor Environments. IEEE J. Sel. Top. Signal Process. 2014, 9, 409-421. [CrossRef]

64. Tan, K.; Cheng, X. Correction of Incidence Angle and Distance Effects on TLS Intensity Data Based on Reference Targets. Remote Sens. 2016, 8, 251. [CrossRef]

65. Shin, J.-I.; Park, H.; Kim, T. Characteristics of Laser Backscattering Intensity to Detect Frozen and Wet Surfaces on Roads. J. Sens. 2019, 2019, 1-9. [CrossRef]

66. Kaasalainen, S.; Kaartinen, H.; Kukko, A. Snow cover change detection with laser scanning range and brightness measurements. EARSeL eProc. 2008, 7, 133-141.

67. Alkadri, M.F.; Turrin, M.; Sariyildiz, S. A computational workflow to analyse material properties and solar radiation of existing contexts from attribute information of point cloud data. Build. Envir. 2019, 155, $268-282$. [CrossRef]

68. Suchocki, C.; Błaszczak-Bak, W. Down-Sampling of Point Clouds for the Technical Diagnostics of Buildings and Structures. Geoscience 2019, 9, 70. [CrossRef]

69. Armesto, J.; Riveiro, B.; González-Aguilera, D.; Rivas-Brea, M.T. Terrestrial laser scanning intensity data applied to damage detection for historical buildings. J. Archaeol. Sci. 2010, 37, 3037-3047. [CrossRef]

70. Soudarissanane, S. The Geometry of Terrestrial Laser Scanning; Identification of Errors, Modeling and Mitigation of Scanning Geometry. Ph.D. Thesis, Faculty of Civil Engineering and Geosciences, TU Delft, Delft, The Netherlands, January 2016.

71. TOPCON. GLS-2000 Series: Multi Functional 3D Laser Scanner. Available online: https: //www.topconpositioning.com/sites/default/files/product_files/gls-2000series_broch_7010-2152_reve_ team_en_us_lores.pdf (accessed on 6 January 2020).

72. Maptek. Maptek I-Site Studio; Maptek: Denver, CO, USA, 2015.

73. Compare, C. Cloud Compare: 3D point cloud and mesh processing software, open source project. 2019. Available online: http://www.cloudcompare.org/ (accessed on 1 April 2020).

74. Matlab. MathWorks. 2020. Available online: https://nl.mathworks.com/products/matlab.html?s_tid=hp_ff_ p_matlab (accessed on 2 April 2020).

75. Rhinoceros. Rhinoceros: design, model, present, realize, analyse...2020. Available online: https://www. rhino3d.com/ (accessed on 4 April 2020).

76. Davidson, Scoot. Grasshopper: Algorithmic modelling for rhino. 2020. Available online: https://www. grasshopper3d.com/ (accessed on 4 April 2020).

77. Ladybug. Ladybug tools. 2020. Available online: https://www.ladybug.tools/ (accessed on 5 April 2020).

78. Boulch, A.; Marlet, R. Deep Learning for Robust Normal Estimation in Unstructured Point Clouds. In Proceedings of the Eurographics Symposium on Geometry Processing, Berlin, Germany, 20-24 June 2016.

79. Sasidharan, S. A Normalization scheme for Terrestrial LiDAR Intensity Data by Range and Incidence Angle. Internatl. J. Emerging Technol. Adv. Eng. 2016, 6, 322-328. 
80. Ministry of Foreign Affairs. Climate change profile: Indonesia; Ministry of Foreign Affairs of The Netherlands: The Hague, The Netherlands, 2018.

81. Optotherm. Optotherm Thermal Imaging, Optotherm Inc. 2018. Available online: https://www.optotherm. com/emiss-examples.htm (accessed on 9 January 2020).

82. Damayanti, H.; Tumiwa, F.; Citraningrum, M. Residential Rooftop Solar: Technical and Market Potential in 34 Provinces in Indonesia; Institute for Essential Services Reform (IESR)-Accelerating Low-Carbon Energy Transition: Jakarta, Indonesia, 2019.

83. Mass, W. What's Next? How Do We Make Vertical Urban Design? In Proceedings of the Council on Tall Buildings and Urban Habitat (CTBUH), Shenzen, China, 16-21 October 2016.

84. MVRDV. Bastide Niel. MVRDV. 2010. Available online: https://www.mvrdv.nl/projects/46/bastide-niel(accessed on 3 August 2020).

(C) 2020 by the authors. Licensee MDPI, Basel, Switzerland. This article is an open access article distributed under the terms and conditions of the Creative Commons Attribution (CC BY) license (http://creativecommons.org/licenses/by/4.0/). 